\title{
Volatile Compositions and Antifungal Activities of Native American Medicinal Plants: Focus on the Asteraceae
}

\author{
Sims K. Lawson ${ }^{1}$, Layla G. Sharp ${ }^{1}$, Chelsea N. Powers ${ }^{1}$, Robert L. McFeeters ${ }^{1}$, Prabodh Satyal ${ }^{2}$ \\ and William N. Setzer $1,2, *$ (D) \\ 1 Department of Chemistry, University of Alabama in Huntsville, Huntsville, AL 35899, USA; \\ sk10003@uah.edu (S.K.L.); lgs0003@uah.edu (L.G.S.); cnp0007@uah.edu (C.N.P.); rlm0004@uah.edu (R.L.M.) \\ 2 Aromatic Plant Research Center, 230 N 1200 E, Suite 100, Lehi, UT 84043, USA; psatyal@aromaticplant.org \\ * Correspondence: wsetzer@chemistry.uah.edu; Tel.: +1-256-824-6519
}

Received: 26 December 2019; Accepted: 16 January 2020; Published: 19 January 2020

check for updates

\begin{abstract}
In the past, Native Americans of North America had an abundant traditional herbal legacy for treating illnesses, disorders, and wounds. Unfortunately, much of the ethnopharmacological knowledge of North American Indians has been lost due to population destruction and displacement from their native lands by European-based settlers. However, there are some sources of Native American ethnobotany remaining. In this work, we have consulted the ethnobotanical literature for members of the Asteraceae used in Cherokee and other Native American traditional medicines that are native to the southeastern United States. The aerial parts of Eupatorium serotinum, Eurybia macrophylla, Eutrochium purpureum, Polymnia canadensis, Rudbeckia laciniata, Silphium integrifolium, Smallanthus uvedalia, Solidago altissima, and Xanthium strumarium were collected from wild-growing plants in north Alabama. The plants were hydrodistilled to obtain the essential oils and the chemical compositions of the essential oils were determined by gas chromatography-mass spectrometry. The essential oils were tested for in-vitro antifungal activity against Aspergillus niger, Candida albicans, and Cryptococcus neoformans. The essential oil of E. serotinum showed noteworthy activity against $C$. neoformans with a minimum inhibitory concentration (MIC) value of $78 \mu \mathrm{g} / \mathrm{mL}$, which can be attributed to the high concentration of cyclocolorenone in the essential oil.
\end{abstract}

Keywords: ethnopharmacology; essential oil; chemical composition; Cryptococcus neoformans; cyclocolorenone

\section{Introduction}

Many aspects of modern medicine have relied on the traditional knowledge of native cultures, including, for example, traditional Indian medicine (Ayurveda) [1], traditional Chinese medicine (TCM) [2], and traditional Islamic medicine [3]. Unfortunately, many of the traditional uses of medicinal plants are being lost due to several reasons. Recent generations are less interested in traditional knowledge, and habitat destruction and forced migration have reduced access to medicinal plants. The Native Americans of North America also had rich traditions of medicinal plant use. However, much of this knowledge has been lost due to population declines and displacement from native lands. Nevertheless, there are still some existing references to the ethnobotanical uses of medicinal plants by Native Americans [4].

Eupatorium serotinum Michx., "late boneset", is native to eastern North America and ranges from Texas, Oklahoma, and Kansas, to the Atlantic coast and from the Gulf of Mexico north to Wisconsin and Michigan [5]. The Houma people of Louisiana used a decoction of the flowers to treat typhoid fever [6]. Extracts of the aerial parts of E. serotinum have yielded germacranolide sesquiterpenoids $[7,8]$. 
Eurybia macrophylla (L.) Cass. (syn. Aster macrophyllus L.), "bigleaf aster", is native to southeastern Canada and northeastern United States, as far south as north Alabama and north Georgia [9]. The Iroquois used a decoction of the roots as a blood medicine and to treat venereal disease; the Ojibwa people ate the leaves of E. macrophylla as a medicine and food [6].

Eutrochium purpureum (L.) E.E. Lamont (syn. Eupatorium purpureum L.), "purple Joe-Pye weed", ranges from central to eastern North America, from the Great Lakes region south to the Gulf of Mexico [10]. The Cherokee used the roots as a treatment for rheumatism, for kidney problems, and for "female problems"; the Chippewa inhaled the vapors from an infusion of the plant for colds; the Navajo used the plant as an antidote for poison; and the Potawatomi people applied a poultice of the leaves on burns [6].

Polymnia canadensis L., "white flower leafcup", is found in eastern North America from Alabama and Georgia north to Ontario, and from Kansas and Oklahoma east to the Appalachians and New York [11]. The Houma people applied a poultice of the crushed leaves to swellings; the Iroquois used the plant to relieve toothache [6]. Extracts of the aerial parts of P. canadensis have yielded diterpenoid carboxylic acids and germacranolides [12].

Rudbeckia laciniata L., "cutleaf coneflower", is widespread in the United States and Canada [13]. There are eight varieties of $R$. laciniata, namely ampla, bipinnata, digitata, gaspereauensis, heterophylla, hortensia, humilis, and laciniata [14]; R. laciniata var. laciniata is the common variety found in eastern North America [15]. The Cherokee ate the cooked greens to "keep well", while the Chippewa applied a poultice of the flowers to treat burns [6]. Several lignans, flavonoid glycosides, and quinic acid derivatives have been isolated from the aerial parts of $R$. laciniata, and sesquiterpenoids have been isolated from root extracts [4].

Silphium integrifolium Michx., "whole-leaf rosinweed", ranges from Wisconsin and Michigan, south through Alabama and Mississippi, and west as far as New Mexico [16]. An infusion of the leaves of S. integrifolium was taken by the Meskwaki people for "bladder troubles" [6]. Flavonoids, oleanolic acid glycosides, and phenolic acids have been identified in the aerial parts of $S$. integrifolium [17].

Smallanthus uvedalia (L.) Mack. (syn. Polymnia uvedalia (L.) L.), "yellow flower leafcup", is found in the southeastern United States from Virginia to Florida, west to eastern Texas and Oklahoma [18]. The Cherokee used the bruised roots on burns and cuts; the Iroquois took an infusion of the shoots and roots to treat back pain and vomiting [6]. The plant is the source of several germacranolide sesquiterpenoids and ent-kaurane diterpenoids [4].

Solidago altissima L. (syn. Solidago canadensis L.), "Canada goldenrod", ranges across most of North America from Canada to northern Mexico [19]. The Okanagan-Colville and the Thompson tribes used an infusion of the roots and shoots of S. altissima to treat fevers [6], and the Cherokee took an infusion of Solidago spp. to treat fevers. The phytochemistry of S. canadensis has been extensively studied and found to contain saponins [20,21], flavonoids [22-24], polyacetylenes [25,26], diterpenoids [27], and triterpenoids [28].

Xanthium strumarium L., "rough cocklebur", ranges throughout North America and is considered a noxious weed in the southeastern United States [29]. The White Mountain Apache tribe took a root decoction to treat fevers; the Mahuma people of Southern California used the plant to treat rheumatism, tuberculosis, and gonorrhea [6]. The aerial parts of X. strumarium contain alkaloids, sesquiterpene lactones (guaianolides, germacranolides, and elemanolides), phenolic compounds, and the toxic carboxylic acid atractyloside, a kaurene glycoside [30].

We have had an interest in the volatile chemistry and biological activity of Native American medicinal plants [31-42], including members of the Asteraceae [43-45]. As part of our continuing investigations, the purpose of this work was to seek out additional species of Asteraceae important in Native American traditional medicine growing wild in northern Alabama and to obtain the essential oils by hydrodistillation of the aerial parts. As a test for biological activity, the essential oils were then screened for antifungal activity against three potentially pathogenic fungal strains. Aspergillus niger, Candida albicans, and Cryptococcus neoformans are the causative agents of opportunistic Aspergillus lung disease, candidiasis, and cryptococcosis, respectively. 


\section{Results and Discussion}

The essential oils from E. serotinum, E. macrophylla, E. purpureum, P. canadensis, R. laciniata, S. integrifolium, S. uvedalia, S. altissima, and X. strumarium were obtained from the fresh aerial parts of the plants by hydrodistillation, generally in low yield. The essential oils were analyzed by GC and GC-MS (Tables 1, 3-9, and 11).

\subsection{Eupatorium serotinum Michx.}

The essential oil from the aerial parts of E. serotinum was rich in sesquiterpenoids, with cyclocolorenone $(23.38 \%)$, germacrene D (6.58\%), and palustrol (5.32\%), along with an unidentified sesquiterpenoid $(5.72 \%)$ as the major components (Table 1$)$.

Table 1. Chemical composition of the essential oil of Eupatorium serotinum Michx.

\begin{tabular}{|c|c|c|c|c|c|c|c|}
\hline $\mathbf{R I}^{\mathbf{a}}$ & $\mathbf{R I}^{\mathbf{b}}$ & Compound & $\% \pm \mathrm{SD}$ & $\mathbf{R I}^{\mathbf{a}}$ & $\mathbf{R I}^{\mathbf{b}}$ & Compound & $\% \pm \mathrm{SD}$ \\
\hline 802 & 801 & Hexanal & $0.16 \pm 0.02$ & 1531 & 1533 & trans-Cadina-1,4-diene & $0.20 \pm 0.07$ \\
\hline 810 & 796 & 2-Hexanol & $0.92 \pm 0.01$ & 1540 & - & Unidentified $^{\mathrm{e}}$ & $1.28 \pm 0.05$ \\
\hline 850 & 846 & $(2 E)$-Hexenal & $0.86 \pm 0.11$ & 1542 & 1539 & $\alpha$-Copaen-11-ol & $7.89 \pm 0.13$ \\
\hline 932 & 932 & $\alpha$-Pinene & $0.17 \pm 0.01$ & 1548 & - & Unidentified $^{\mathrm{f}}$ & $1.76 \pm 0.04$ \\
\hline 949 & 946 & Camphene & $1.78 \pm 0.02$ & 1550 & - & Unidentified $\mathrm{g}$ & $0.75 \pm 0.03$ \\
\hline 977 & 974 & $\beta$-Pinene & $0.18 \pm 0.02$ & 1558 & 1559 & Germacrene B & $0.44 \pm 0.03$ \\
\hline 999 & 1001 & $\delta$-2-Carene & $0.15 \pm 0.01$ & 1562 & - & Eudesmenol $^{\mathrm{h}}$ & $0.32 \pm 0.09$ \\
\hline 1029 & 1024 & Limonene & $0.26 \pm 0.01$ & 1569 & 1567 & Palustrol & $5.32 \pm 0.12$ \\
\hline 1283 & 1287 & Bornyl acetate & $4.72 \pm 0.07$ & 1575 & 1574 & Germacra-1(10),5-dien- $4 \beta$-ol & $0.91 \pm 1.10$ \\
\hline 1326 & - & Unidentified $^{c}$ & $0.93 \pm 0.03$ & 1581 & 1577 & Spathulenol & $1.58 \pm 0.83$ \\
\hline 1346 & 1345 & $\alpha$-Cubebene & $0.59 \pm 0.01$ & 1588 & 1590 & Globulol & $0.58 \pm 0.03$ \\
\hline 1375 & 1374 & $\alpha$-Copaene & $0.11 \pm 0.05$ & 1593 & 1592 & Viridiflorol & $1.12 \pm 0.09$ \\
\hline 1383 & 1387 & $\beta$-Bourbonene & $0.06 \pm 0.01$ & 1596 & - & Unidentified $^{\mathrm{i}}$ & $1.20 \pm 0.02$ \\
\hline 1397 & 1387 & $\beta$-Cubebene & $3.65 \pm 0.09$ & 1603 & 1602 & Ledol & $2.80 \pm 0.03$ \\
\hline 1406 & 1409 & $\alpha$-Gurjunene & $0.74 \pm 0.02$ & 1620 & 1611 & Germacra-1(10),5-dien- $4 \alpha$-ol & $1.44 \pm 0.13$ \\
\hline 1417 & 1419 & $\beta$-Ylangene & $0.09 \pm 0.03$ & 1622 & 1624 & Selina-6-en- $4 \beta$-ol & $0.31 \pm 0.03$ \\
\hline 1418 & 1417 & $\beta$-Caryophyllene & $0.96 \pm 0.01$ & 1627 & 1627 & 1-epi-Cubenol & $0.61 \pm 0.10$ \\
\hline 1428 & 1434 & $\gamma$-Elemene & $0.28 \pm 0.05$ & 1638 & 1639 & cis-Guaia-3,9-dien-11-ol & $0.12 \pm 0.01$ \\
\hline 1448 & 1448 & cis-Muurola-3,5-diene & $0.07 \pm 0.03$ & 1642 & 1638 & $\tau$-Cadinol & $0.80 \pm 0.03$ \\
\hline 1455 & 1452 & $\alpha$-Humulene & $0.41 \pm 0.04$ & 1642 & 1640 & $\tau$-Muurolol & $0.62 \pm 0.08$ \\
\hline 1471 & 1475 & trans-Cadina-1(6),4-diene & $0.25 \pm 0.03$ & 1646 & 1644 & $\alpha$-Muurolol (= $\delta$-Cadinol) & $0.69 \pm 0.07$ \\
\hline 1480 & 1484 & Germacrene D & $6.58 \pm 0.09$ & 1648 & 1646 & Agarospirol II & $1.10 \pm 0.04$ \\
\hline 1486 & 1488 & $\delta$-Selinene & $0.27 \pm 0.02$ & 1654 & 1652 & $\alpha$-Cadinol & $2.31 \pm 0.04$ \\
\hline 1488 & 1489 & $\beta$-Selinene & $0.17 \pm 0.01$ & 1668 & - & Unidentified ${ }^{j}$ & $5.72 \pm 0.14$ \\
\hline 1491 & 1493 & trans-Muurola-4(14),5-diene & $0.69 \pm 0.03$ & 1751 & 1759 & Cyclocolorenone & $23.38 \pm 0.43$ \\
\hline 1494 & 1493 & epi-Cubebol & $1.81 \pm 0.03$ & & & Green leaf volatiles & 1.94 \\
\hline 1497 & 1500 & $\alpha$-Muurolene & $0.34 \pm 0.02$ & & & Monoterpene hydrocarbons & 2.53 \\
\hline 1502 & - & Unidentified $^{\mathrm{d}}$ & $1.30 \pm 0.02$ & & & Oxygenated monoterpenoids & 4.72 \\
\hline 1512 & 1513 & $\gamma$-Cadinene & $0.21 \pm 0.00$ & & & Sesquiterpene hydrocarbons & 19.14 \\
\hline 1514 & 1514 & Cubebol & $4.18 \pm 0.11$ & & & Oxygenated sesquiterpenoids & 57.90 \\
\hline 1517 & 1522 & $\delta$-Cadinene & $3.02 \pm 0.25$ & & & Total Identified & 86.23 \\
\hline
\end{tabular}

${ }^{\mathrm{a}} \mathrm{RI}=$ Retention index determined in reference to a homologous series of $n$-alkanes on a ZB-5ms column. ${ }^{\mathrm{b}} \mathrm{RI}$ values from the databases. ${ }^{\mathrm{c}} \mathrm{MS}(\mathrm{EI})$ (mass spectrum (electron impact)): 162(84\%), 147(96\%), 133(20\%), 120(32\%), 119(41\%), 108(35\%), 105(100\%), 91(63\%), 79(29\%), 77(22\%), 55(11\%), 53(12\%), 41(14\%). ${ }^{\mathrm{d}} \mathrm{MS}(\mathrm{EI}):$ 202(7\%), 187(9\%), 162(68\%), $159(31 \%), 147(50 \%), 145(32 \%), 132(49 \%), 119(66 \%), 105(89 \%), 91(48 \%), 81(18 \%), 79(20 \%), 77(16 \%), 59(100 \%), 43(20 \%)$, 41(20\%). e MS(EI): 202(4\%), 187(13\%), 162(56\%), 159(59\%), 147(39\%), 145(40\%), 132(73\%), 131(39\%), 119(73\%), $106(48 \%), 105(88 \%), 91(47 \%), 81(16 \%), 79(25 \%), 77(19 \%), 59(100 \%), 55(18 \%), 43(19 \%), 41(20 \%) .{ }^{f} \mathrm{MS}(\mathrm{EI}): 220(24 \%)$, 205(17\%), 163(19\%), 120(35\%), 110(100\%), 105(20\%), 95(35\%), 69(44\%), 55(20\%), 41(24\%). g MS(EI): 220(47\%), $163(25 \%), 161(32 \%), 121(100 \%), 108(42 \%), 93(42 \%), 81(59 \%), 69(17 \%), 55(15 \%), 41(18 \%) .{ }^{\mathrm{h}}$ Correct isomer not identified. ${ }^{\mathrm{i}} \mathrm{MS}(\mathrm{EI}):$ 220(49\%), 205(7\%), 163(33\%), 161(28\%), 121(100\%), 108(40\%), 93(35\%), 81(80\%), 69(20\%), 55(17\%), 41(19\%). ${ }^{j} \mathrm{MS}(\mathrm{EI}): 202(46 \%), 187(67 \%), 174(40 \%), 162(60 \%), 159(100 \%), 147(89 \%), 134(30 \%), 131(23 \%)$, $119(62 \%), 105(71 \%), 91(50 \%), 59(61 \%), 43(20 \%), 41(22 \%)$.

To our knowledge, there have been no previous reports on the essential oil composition of E. serotinum. The phytochemistry of the genus Eupatorium has been reviewed [46] and there have been numerous reports on the essential oil compositions from other species of the genus (Table 2). There is much variability in the essential oil compositions of Eupatorium species, both between species and within species. Nevertheless, sesquiterpenoids often dominate the essential oils of Eupatorium species. 
Table 2. Major components and biological activities of Eupatorium essential oils

\begin{tabular}{|c|c|c|c|c|}
\hline Eupatorium spp. Essential Oil & Location & Major Components & Biological Activity & Ref. \\
\hline E. adenophorum (aerial parts) & Nainital, India & $\begin{array}{l}\text { camphene }(8.9 \%), p \text {-cymene }(16.6 \%) \text {, bornyl acetate }(15.6 \%) \text {, amorph-4-en-7-ol }(9.6 \%) \text {, } \\
\alpha \text {-cadinol }(6.2 \%) \text {, amorpha- } 4,7(11) \text {-dien- } 8 \text {-one }(7.8 \%)\end{array}$ & none reported & [47] \\
\hline E. adenophorum (leaves) & Palampur, India & $\begin{array}{l}\text { bornyl acetate }(9.0 \%) \text {, germacrene D }(5.7 \%), \beta \text {-bisabolene }(6.2 \%), 1 \text {-naphthalenol } \\
(17.5 \%), \alpha \text {-bisabolol }(9.5 \%)\end{array}$ & Antibacterial (Rhodococcus rhodochrous, MBC $12.5 \mu \mathrm{L} / \mathrm{mL}$ ) & [48] \\
\hline E. adenophorum (twigs) & Uttar Pradesh, India & $\begin{array}{l}\text { camphene }(12.1 \%), \alpha \text {-phellandrene }(8.6 \%), \alpha \text {-terpinene }(6.5 \%) \text {, } p \text {-cymene }(11.6 \%) \text {, } \\
\text { bornyl acetate }(10.6 \%) \text {, acoradiene }(10.1 \%), \alpha \text {-bisabolol }(5.3 \%)\end{array}$ & $\begin{array}{l}\text { Antibacterial (Erwinia herbicola, MIC } 0.25 \mu \mathrm{L} / \mathrm{mL} \text {; } \\
\text { Pseudomonas putida, MIC } 2.0 \mu \mathrm{L} / \mathrm{mL} \text { ) }\end{array}$ & [49] \\
\hline E. adenophorum (inflorescence) & Palampur, India & $\begin{array}{l}\text { bornyl acetate (6.3\%), } \beta \text {-caryophyllene ( } 5.4 \%), \gamma \text {-muurolene }(11.7 \%), \gamma \text {-curcumene } \\
(5.7 \%), \gamma \text {-cadinene }(18.4 \%), 3 \text {-acetoxyamorpha-4,7(11)-dien-8-one }(7.4 \%)\end{array}$ & $\begin{array}{l}\text { Antifungal (Macrophomina phaseolina, } \mathrm{EC}_{50} 0.076 \mu \mathrm{L} / \mathrm{mL} ; \\
\text { Rhizoctonia solani, } \mathrm{EC}_{50} 0.094 \mu \mathrm{L} / \mathrm{mL} ; \text { Fusarium oxysporum, } \\
\mathrm{EC}_{50} 0.120 \mu \mathrm{L} / \mathrm{mL} \text { ) }\end{array}$ & [50] \\
\hline E. amygdalinum (aerial parts) & Amapá, Brazil & $\begin{array}{l}\beta \text {-cubebene }(5.7 \%), \beta \text {-caryophyllene }(12.3 \%) \text {, germacrene D }(15.5 \%), \delta \text {-cadinene }(5.8 \%) \text {, } \\
\text { caryophyllene oxide }(17.4 \%)\end{array}$ & none reported & [51] \\
\hline E. argentinum (leaves) & Córdoba, Argentina & $\begin{array}{l}\alpha \text {-pinene }(17.0 \%), \beta \text {-pinene }(6.1 \%), p \text {-cymene }(12.5 \%) \text {, thymyl acetate }(9.7 \%), \\
\beta \text {-caryophyllene }(7.2 \%)\end{array}$ & none reported & [52] \\
\hline E. arnottianum (aerial parts) & Córdoba, Argentina & $\begin{array}{l}\alpha \text {-pinene }(13.7 \%), p \text {-cymene }(30.0 \%), \beta \text {-ocimene }(5.3 \%) \text {, thymyl acetate }(12.3 \%) \text {, } \\
\beta \text {-caryophyllene }(11.7 \%)\end{array}$ & none reported & [53] \\
\hline E. arnottianum (aerial parts) & Córdoba, Argentina & $\begin{array}{l}\text { limonene (32.7\%), piperitenone }(21.2 \%) \text {, trans-dihydrocarvone }(10.2 \%) \text {, camphor } \\
(6.8 \%) \text {, cis-dihydrocarvone }(6.7 \%)\end{array}$ & Antiviral (HSV-1, IC $5052.1 \mu \mathrm{g} / \mathrm{mL}$; DENV-2, $\mathrm{IC}_{50} 38.2 \mu \mathrm{g} / \mathrm{mL}$ ) & [54] \\
\hline E. arnottii (aerial parts) & San Luis, Argentina & $\begin{array}{l}\text { B-caryophyllene }(7.9 \%), \gamma \text {-elemene }(5.9 \%) \text {, germacrene D }(9.8 \%) \text {, cadinene }(5.8 \%) \text {, } \\
\text { spathulenol }(10.6 \%) \text {, phytol }(8.1 \%)\end{array}$ & Insecticidal (Tribolium castaneum, $\mathrm{ED}_{50} 0.15 \mathrm{mg} / \mathrm{cm} 2$ ) & [55] \\
\hline E. ballotaefolium (aerial parts) & Ceará, Brazil & $\begin{array}{l}\alpha \text {-pinene }(6.2 \%) \text {, sabinene }(6.5 \%), \beta \text {-pinene }(5.4 \%) \text {, myrcene }(7.3 \%) \text {, limonene }(15.3 \%) \text {, } \\
(E) \text { - } \beta \text {-ocimene }(10.5 \%), \beta \text {-caryophyllene }(7.5 \%)\end{array}$ & none reported & [56] \\
\hline E. betonicaeforme (leaves) & Ceará, Brazil & $\begin{array}{l}\beta \text {-caryophyllene }(36.1 \%), \alpha \text {-humulene }(13.3 \%), \gamma \text {-muurolene }(20.3 \%), \\
\text { bicyclogermacrene }(15.0 \%)\end{array}$ & Larvicidal (Aedes aegypti, LC 50129 mg/mL) & [57] \\
\hline E. buniifolium (aerial parts) & Canelones, Uruguay & $\alpha$-pinene $(14.7 \%), \beta$-elemene $(12.2 \%)$, germacrene $\mathrm{D}(11.5 \%)$, trans- $\beta$-guaiene $(6.5 \%)$ & none reported & [58] \\
\hline E. buniifolium (aerial parts) & San Luis, Argentina & $\alpha$-pinene $(51.0 \%)$, sabinene $(7.5 \%)$, limonene $(9.6 \%), \beta$-caryophyllene $(5.2 \%)$ & Insecticidal (Tribolium castaneum, $\mathrm{ED}_{50} 0.15 \mathrm{mg} / \mathrm{cm}^{2}$ ) & [55] \\
\hline E. buniifolium (leaves) & Canelones, Uruguay & $\alpha$-pinene $(8.2 \%)$, germacrene $D(11.1 \%)$, trans- $\beta$-guaiene $(7.4 \%)$ & Varroacide (Varroa destructor, $\mathrm{LD}_{99} 0.3 \mathrm{mg} / \mathrm{mL}$ ) & [59] \\
\hline $\begin{array}{l}\text { E. cannabinum ssp. cannabinum } \\
\text { (aerial parts) }\end{array}$ & Agerola, Italy & $\delta$-2-carene $(6.5 \%)$, germacrene D $(33.5 \%), \alpha$-farnesene $(12.9 \%)$ & $\begin{array}{l}\text { Antibacterial (Staphylococcus aureus, Streptococcus fecalis, } \\
\text { Bacillus subtilis, Bacillus cereus, MIC } 1.25 \mathrm{mg} / \mathrm{mL} \text { ) }\end{array}$ & [60] \\
\hline E. cannabinum (leaves) & Tuscany, Italy & thymol methyl ether $(7.8 \%)$, germacrene D $(29.2 \%)$, spathulenol $(7.3 \%)$ & none reported & [61] \\
\hline $\begin{array}{l}\text { E. cannabinum ssp. corsicum } \\
\text { (aerial parts) }\end{array}$ & Corsica, France & $\alpha$-phellandrene $(19.0 \%)$, $p$-cymene $(5.2 \%)$, germacrene $\mathrm{D}(28.5 \%)$ & none reported & [62] \\
\hline E. cannabinum (aerial parts) & Mazandaran, Iran & $\alpha$-terpinene $(17.8 \%)$, thymol methyl ether $(5.2 \%)$, germacrene $\mathrm{D}(9.1 \%)$ & none reported & [63] \\
\hline E. cannabinum (leaves) & Vilnius, Lithuania & $\begin{array}{l}\text { thymol methyl ether }(5.7 \%) \text {, neryl acetate }(9.4 \%) \text {, germacrene D }(11.3 \%) \text {, } \\
\beta \text {-bisabolene }(6.7 \%)\end{array}$ & none reported & [64] \\
\hline E. capillifolium (aerial parts) & Cuba & $\begin{array}{l}p \text {-cymene }(23.7 \%) \text {, thymol methyl ether }(8.9 \%), \beta \text {-bisabolene }(8.2 \%) \text {, } \\
\text { selin-11-en- } 4 \alpha \text {-ol }(12.3 \%)\end{array}$ & none reported & [65] \\
\hline E. capillifolium (aerial parts) & Mississippi, USA & $\begin{array}{l}\text { myrcene }(15.7 \%), \alpha \text {-phellandrene }(6.5 \%) \text {, thymol methyl ether }(36.3 \%) \text {, } \\
\text { 2,5-dimethoxy-p-cymene }(20.8 \%)\end{array}$ & Insecticidal (Stephanitis pyrioides, $\mathrm{LC}_{50} 5800 \mu \mathrm{gg} / \mathrm{mL}$ ) & [66] \\
\hline
\end{tabular}


Table 2. Cont.

\begin{tabular}{|c|c|c|c|c|}
\hline Eupatorium spp. Essential Oil & Location & Major Components & Biological Activity & Ref. \\
\hline E. catarium (aerial parts) & Córdoba, Argentina & $\begin{array}{l}\text { spathulenol (15.5\%), } \beta \text {-caryophyllene (7.8\%), germacrene D ( } 5.5 \%) \text {, } \\
\text { bicyclogermacrene }(5.1 \%)\end{array}$ & Antiviral (HSV-1, $\mathrm{IC}_{50} 47.9 \mu \mathrm{g} / \mathrm{mL}$; DENV-2, IC $5057.3 \mu \mathrm{g} / \mathrm{mL}$ ) & [54] \\
\hline E. conyzoides (aerial parts) & Tocantins, Brazil & $\begin{array}{l}\text { } \text {-caryophyllene }(7.1 \%), \alpha \text {-humulene }(6.6 \%) \text {, germacrene D }(16.8 \%) \text {, } \\
\text { bicyclogermacrene }(7.2 \%) \text {, spathulenol }(8.3 \%)\end{array}$ & none reported & [51] \\
\hline E. glabratum (leaves) & Michoacán, México & $\alpha$-pinene $(29.5 \%), \beta$-pinene $(6.3 \%), \alpha$-phellandrene $(19.6 \%)$ & Insecticidal (Sitophilus zeamais, $\mathrm{LC}_{50} 18.0 \mu \mathrm{L} / \mathrm{mL}$ ) & [67] \\
\hline E. hecatanthum (leaves) & Córdoba, Argentina & $\begin{array}{l}\alpha \text {-pinene }(13.4 \%), \beta \text {-pinene }(7.8 \%), \beta \text {-ocimene }(6.2 \%) \text {, carvacrol }(7.1 \%) \text {, thymyl acetate } \\
(10.6 \%), \beta \text {-caryophyllene }(8.1 \%)\end{array}$ & none reported & [52] \\
\hline E. inulaefolium (aerial parts) & San Luis, Argentina & $\begin{array}{l}\text { limonene }(9.7 \%), \delta \text {-elemene }(10.6 \%), \beta \text {-caryophyllene }(27.7 \%) \text {, } \alpha \text {-humulene }(5.9 \%) \text {, } \\
\text { patchoulene }(9.2 \%) \text {, germacrene D }(13.7 \%) \text {, viridiflorol }(9.2 \%)\end{array}$ & Insecticidal (Tribolium castaneum, $\mathrm{ED}_{50} 0.15 \mathrm{mg} / \mathrm{cm}^{2}$ ) & [55] \\
\hline E. laevigatum (aerial parts) & Roraima, Brazil & $\begin{array}{l}\text { germacrene D (8.6\%), selina-3,7(11)-diene (6.1\%), spathulenol (5.4\%), globulol (16.2\%), } \\
\text { laevigatin }(23.6 \%)\end{array}$ & none reported & [51] \\
\hline E. laevigatum (leaves) & Rio Grande do Sul, Brazil & germacrene D (11.7\%), bicyclogermacrene $(9.3 \%)$, laevigatin $(59.6 \%)$ & none reported & [68] \\
\hline E. macrophyllum (aerial parts) & Chapada dos Guimarães, Brazil & sabinene $(46.7 \%)$, limonene $(23.3 \%)$ & none reported & [51] \\
\hline E. marginatum (aerial parts & Ananindeua, Pará, Brazil & $\begin{array}{l}\text { ar-curcumene }(6.8 \%), \alpha \text {-zingiberene }(57.5 \%), \beta \text {-sesquiphellandrene }(7.1 \%), \\
(E) \text { - } \gamma \text {-bisabolene }(9.7 \%)\end{array}$ & none reported & [51] \\
\hline E. marginatum (aerial parts & Roraima, Brazil & $\alpha$-gurjunene $(19.5 \%)$, germacrene $\mathrm{D}(14.8 \%), \alpha$-selinene $(9.0 \%),(\mathrm{E})-\gamma$-bisabolene $(5.0 \%)$ & none reported & [51] \\
\hline E. odoratum (aerial parts) & Thitsanulok, Thailand & $\begin{array}{l}\alpha \text {-pinene }(8.4 \%), \beta \text {-pinene }(5.6 \%) \text {, pregeijerene }(17.6 \%) \text {, germacrene } \mathrm{D}(11.1 \%) \text {, } \\
\beta \text {-caryophyllene }(7.3 \%) \text {, vestitenone }(6.5 \%)\end{array}$ & none reported & [69] \\
\hline E. odoratum (leaves) & Lagos, Nigeria & $\begin{array}{l}\alpha \text {-pinene }(42.2 \%), \beta \text {-pinene }(10.6 \%), \beta \text {-caryophyllene }(5.4 \%) \text {, germacrene D }(9.7 \%) \text {, } \\
\beta \text {-copaen- } 4 \alpha \text {-ol }(9.4 \%)\end{array}$ & $\begin{array}{l}\text { Antibacterial (Bacillus cereus, MIC } 39 \mu \mathrm{g} / \mathrm{mL} \text { ), antifungal } \\
\text { (Aspergillus niger, MIC } 78 \mu \mathrm{g} / \mathrm{mL} \text { ) }\end{array}$ & [70] \\
\hline E. odoratum (aerial parts) & Western Ghats, India & cis-sabinene hydrate (5.7\%), pregeijerene $(14.2 \%)$, epi-cubebol $(9.8 \%)$, cubebol $(8.6 \%)$ & none reported & [71] \\
\hline E. squalidum (aerial parts) & Amapá, Brazil & $\begin{array}{l}\beta \text {-caryophyllene }(6.2 \%) \text {, germacrene D }(21.6 \%) \text {, bicyclogermacrene }(6.0 \%) \text {, spathulenol } \\
(14.2 \%) \text {, globulol }(25.1 \%)\end{array}$ & none reported & [51] \\
\hline E. squalidum (aerial parts) & Tocantins, Brazil & $\begin{array}{l}\text { limonene }(6.6 \%), \beta \text {-caryophyllene }(9.6 \%) \text {, germacrene D }(10.4 \%) \text {, } \\
\text { caryophyllene oxide }(30.1 \%)\end{array}$ & none reported & [51] \\
\hline E. subhastatum (leaves) & Córdoba, Argentina & $\begin{array}{l}\alpha \text {-pinene }(11.0 \%), \beta \text {-pinene }(5.9 \%), p \text {-cymene }(24.8 \%), \alpha \text {-copaene }(5.1 \%), \\
\alpha \text {-humulene }(5.1 \%)\end{array}$ & none reported & [52] \\
\hline E. triplinerve (leaves) & Lucknow, India & ס-elemene $(5.9 \%), \beta$-caryophyllene $(14.7 \%)$, selina- $4(15), 7(11)$-dien-8-one & none reported & [72] \\
\hline E. viscidum (aerial parts) & San Luis, Argentina & 6-methyl-5-hepten-2-one (18.2\%), spathulenol $(25.2 \%)$ & Insecticidal (Tribolium castaneum, $\mathrm{ED}_{50}>0.212 \mathrm{mg} / \mathrm{cm}^{2}$ ) & [55] \\
\hline
\end{tabular}




\subsection{Eurybia macrophylla (L.) Cass.}

Monoterpene hydrocarbons, limonene (28.66\%), $\beta$-pinene $(8.57 \%)$, and terpinolene $(5.35 \%)$, and germacrane sesquiterpenes, germacrene D $(19.81 \%)$, and germacrene B $(7.07 \%)$, were the major components in the essential oil of E. macrophylla (Table 3). To our knowledge, there are no reports on essential oil compositions of any Eurybia species.

Table 3. Chemical composition of the essential oil of Eurybia macrophylla (L.) Cass.

\begin{tabular}{|c|c|c|c|c|c|c|c|}
\hline $\mathrm{RI}^{\mathrm{a}}$ & $\mathbf{R I}^{\mathrm{b}}$ & Compound & $\% \pm \mathrm{SD}$ & $\mathbf{R I}^{\mathbf{a}}$ & $\mathbf{R I}^{\mathbf{b}}$ & Compound & $\% \pm \mathrm{SD}$ \\
\hline 801 & 797 & (3Z)-Hexenal & $0.06 \pm 0.01$ & 1387 & 1389 & $\beta$-Elemene & $1.48 \pm 0.04$ \\
\hline 802 & 801 & Hexanal & $0.31 \pm 0.05$ & 1418 & 1417 & $\beta$-Caryophyllene & $4.60 \pm 0.07$ \\
\hline 850 & 846 & (2E)-Hexenal & $1.44 \pm 0.06$ & 1427 & 1434 & $\gamma$-Elemene & $3.16 \pm 0.01$ \\
\hline 924 & 924 & $\alpha$-Thujene & $0.16 \pm 0.01$ & 1454 & 1452 & $\alpha$-Humulene & $0.64 \pm 0.01$ \\
\hline 932 & 974 & $\alpha$-Pinene & $3.12 \pm 0.04$ & 1473 & 1471 & Massoia lactone & $0.35 \pm 0.04$ \\
\hline 948 & 946 & Camphene & $0.60 \pm 0.00$ & 1480 & 1484 & Germacrene D & $19.81 \pm 0.20$ \\
\hline 971 & 969 & Sabinene & $0.15 \pm 0.02$ & 1487 & 1489 & $\beta$-Selinene & $0.31 \pm 0.05$ \\
\hline 977 & 974 & $\beta$-Pinene & $8.57 \pm 0.07$ & 1494 & 1500 & Bicyclogermacrene & $1.95 \pm 0.02$ \\
\hline 988 & 988 & Myrcene & $1.79 \pm 0.02$ & 1497 & 1500 & $\alpha$-Muurolene & $0.16 \pm 0.03$ \\
\hline 989 & 988 & Dehydro-1,8-cineole & $0.28 \pm 0.01$ & 1516 & 1522 & $\delta$-Cadinene & $0.19 \pm 0.02$ \\
\hline 1006 & 1002 & $\alpha$-Phellandrene & $0.88 \pm 0.01$ & 1536 & 1537 & $\alpha$-Cadinene & $0.10 \pm 0.02$ \\
\hline 1016 & 1014 & $\alpha$-Terpinene & $0.16 \pm 0.02$ & 1557 & 1559 & Germacrene B & $7.07 \pm 0.07$ \\
\hline 1024 & 1020 & $p$-Cymene & $0.15 \pm 0.01$ & 1575 & 1577 & Spathulenol & $0.10 \pm 0.01$ \\
\hline 1028 & 1024 & Limonene & $28.66 \pm 0.33$ & 1581 & 1582 & Caryophyllene oxide & $0.38 \pm 0.01$ \\
\hline 1030 & 1025 & $\beta$-Phellandrene & $0.75 \pm 0.03$ & 1595 & 1592 & Viridiflorol & $0.23 \pm 0.03$ \\
\hline 1034 & 1032 & (Z)- $\beta$-Ocimene & $0.18 \pm 0.00$ & 1627 & 1629 & iso-Spathulenol & $0.08 \pm 0.01$ \\
\hline 1044 & 1044 & (E)- $\beta$-Ocimene & $2.14 \pm 0.02$ & 1641 & 1638 & $\tau$-Cadinol & $0.09 \pm 0.02$ \\
\hline 1057 & 1054 & $\gamma$-Terpinene & $0.37 \pm 0.01$ & 1643 & 1640 & $\tau$-Murrolol & $0.12 \pm 0.03$ \\
\hline 1084 & 1086 & Terpinolene & $5.35 \pm 0.08$ & 1646 & 1644 & $\alpha$-Muurolol (= $\delta$-Cadinol) & $0.10 \pm 0.01$ \\
\hline 1112 & 1114 & (E)-4,8-Dimethylnona-1,3,7-triene & $0.35 \pm 0.01$ & 1654 & 1652 & $\alpha$-Cadinol & $0.47 \pm 0.02$ \\
\hline 1124 & 1118 & cis-p-Menth-2-en-1-ol & $0.72 \pm 0.01$ & 1832 & 1835 & Neophytadiene & $0.05 \pm 0.02$ \\
\hline 1142 & 1136 & trans-p-Menth-2-en-1-ol & $0.44 \pm 0.01$ & 1838 & 1841 & Phytone & $0.05 \pm 0.02$ \\
\hline 1187 & 1179 & p-Cymen-8-ol & $0.21 \pm 0.03$ & & & Green leaf volatiles & 1.91 \\
\hline 1195 & 1186 & $\alpha$-Terpineol & $0.06 \pm 0.02$ & & & Monoterpene hydrocarbons & 53.03 \\
\hline 1197 & 1195 & cis-Piperitol & $0.15 \pm 0.02$ & & & Oxygenated monoterpenoids & 2.30 \\
\hline 1209 & 1207 & trans-Piperitol & $0.17 \pm 0.01$ & & & Sesquiterpene hydrocarbons & 40.03 \\
\hline 1283 & 1287 & Bornyl acetate & $0.27 \pm 0.10$ & & & Oxygenated sesquiterpenoids & 1.59 \\
\hline 1292 & 1293 & Undecan-2-one & $0.05 \pm 0.01$ & & & Diterpenoids & 0.11 \\
\hline \multirow[t]{2}{*}{1333} & 1335 & $\delta$-Elemene & $0.50 \pm 0.00$ & & & Others & 0.75 \\
\hline & & & & & & Total Identified & 99.72 \\
\hline
\end{tabular}

${ }^{\mathrm{a}} \mathrm{RI}=$ Retention index determined in reference to a homologous series of $n$-alkanes on a ZB- $5 \mathrm{~ms}$ column. ${ }^{\mathrm{b}} \mathrm{RI}$ values from the databases.

\subsection{Eutrochium purpureum (L.) E.E. Lamont (syn. Eupatorium purpureum L.)}

The major components in the essential oil of E. purpureum were the green leaf volatiles (2E)-hexenal $(60.59 \%)$ and hexanal $(6.78 \%)$, along with the aromatic compounds eugenol (11.68\%) and methyl salicylate (10.31\%; Table 4). There have apparently been no previous reports on the essential oil composition of E. purpureum or any other Eutrochium species. There are numerous reports on Eupatorium essential oils, however (see above).

Table 4. Chemical composition of the essential oil of Eutrochium purpureum (L.) E.E. Lamont.

\begin{tabular}{|c|c|c|c|c|c|c|c|}
\hline $\mathrm{RI}^{\mathrm{a}}$ & $\mathbf{R I}^{\mathbf{b}}$ & Compound & $\% \pm \mathrm{SD}$ & $\mathbf{R I}^{\mathbf{a}}$ & $\mathbf{R I}^{b}$ & Compound & $\% \pm \mathrm{SD}$ \\
\hline 801 & 797 & (3Z)-Hexenal & $1.01 \pm 0.11$ & 1206 & 1201 & Decanal & $0.37 \pm 0.05$ \\
\hline 802 & 801 & Hexanal & $6.78 \pm 0.17$ & 1351 & 1356 & Eugenol & $11.68 \pm 0.14$ \\
\hline 850 & 946 & $(2 E)$-Hexenal & $60.59 \pm 1.00$ & 1417 & 1417 & $\beta$-Caryophyllene & $0.24 \pm 0.02$ \\
\hline 865 & 963 & 1-Hexanol & $2.35 \pm 0.41$ & 1479 & 1484 & Germacrene D & $0.67 \pm 0.10$ \\
\hline 931 & 932 & $\alpha$-Pinene & $1.48 \pm 0.09$ & 1559 & 1561 & (E)-Nerolidol & $0.50 \pm 0.01$ \\
\hline 943 & - & Unidentified $^{c}$ & $0.56 \pm 0.07$ & & & Green leaf volatiles & 71.47 \\
\hline 1004 & 998 & Octanal & $0.33 \pm 0.04$ & & & Monoterpene hydrocarbons & 2.36 \\
\hline 1005 & 1004 & (3Z)-Hexenyl acetate & $0.72 \pm 0.12$ & & & Sesquiterpene hydrocarbons & 0.91 \\
\hline 1028 & 1024 & Limonene & $0.88 \pm 0.07$ & & & Oxygenated sesquiterpenoids & 0.50 \\
\hline 1045 & 1036 & Benzene acetaldehyde & $0.60 \pm 0.03$ & & & Benzenoids & 22.59 \\
\hline 1105 & 1100 & Nonanal & $0.91 \pm 0.19$ & & & Fatty aldehydes & 1.61 \\
\hline 1192 & 1190 & Methyl salicylate & $10.31 \pm 0.18$ & & & Total Identified & 99.44 \\
\hline
\end{tabular}

${ }^{\mathrm{a}} \mathrm{RI}=$ Retention index determined in reference to a homologous series of $n$-alkanes on a ZB- $5 \mathrm{~ms}$ column. ${ }^{\mathrm{b}} \mathrm{RI}$ values from the databases. ${ }^{c} \mathrm{MS}(\mathrm{EI}):$ 208(8\%), 97(100\%), 96(17\%), 86(9\%), 69(12\%), 56(22\%), 55(64\%), 43(18\%). 


\subsection{Polymnia canadensis L.}

$\alpha$-Phellandrene (28.30\%), $\alpha$-pinene (19.71\%), and germacrene D $(11.42 \%)$ were the major components in the essential oil from the aerial parts of $P$. canadensis (Table 5). The volatile chemical profile of $P$. canadensis in this current work is in marked contrast to our previous report on this species [45]. Previous samples were rich in the sesquiterpene hydrocarbons germacrene D (63.6\% and $44.5 \%)$ and $\beta$-caryophyllene (15.9\% and $14.8 \%$ ). The differences in compositions are likely due to seasonal variation (the current sample was collected in July, 2018, while the previous samples were collected in September, 2015, and December, 2016, respectively). We cannot rule out, however, chemical profile differences attributable to environmental differences or biotic differences (e.g., genetics, herbivory, or pathogen stress).

Table 5. Chemical composition of the essential oil of Polymnia canadensis L.

\begin{tabular}{|c|c|c|c|c|c|c|c|}
\hline $\mathrm{RI}^{\mathrm{a}}$ & $\mathrm{RI}^{\mathrm{b}}$ & Compound & $\% \pm \mathrm{SD}$ & $\mathrm{RI}^{\mathrm{a}}$ & $\mathbf{R I}^{\mathrm{b}}$ & Compound & $\% \pm \mathrm{SD}$ \\
\hline 802 & 801 & Hexanal & $0.28 \pm 0.04$ & 1417 & 1417 & $\beta$-Caryophyllene & $3.05 \pm 0.02$ \\
\hline 811 & 796 & 2-Hexanol & $0.17 \pm 0.01$ & 1428 & 1430 & $\beta$-Copaene & $0.09 \pm 0.02$ \\
\hline 850 & 850 & (3Z)-Hexenol & $4.31 \pm 0.16$ & 1446 & 1453 & Geranyl acetone & $0.17 \pm 0.01$ \\
\hline 861 & 854 & (2E)-Hexenol & $0.08 \pm 0.01$ & 1454 & 1452 & $\alpha$-Humulene & $1.14 \pm 0.00$ \\
\hline 864 & 863 & 1-Hexanol & $0.30 \pm 0.02$ & 1458 & 1458 & allo-Aromadendrene & $0.17 \pm 0.02$ \\
\hline 921 & 921 & Tricyclene & $0.07 \pm 0.01$ & 1479 & 1484 & Germacrene D & $11.42 \pm 0.01$ \\
\hline 924 & 924 & $\alpha$-Thujene & $0.06 \pm 0.01$ & 1484 & 1486 & Phenylethyl 2-methylbutanoate & $0.18 \pm 0.04$ \\
\hline 932 & 932 & $\alpha$-Pinene & $19.71 \pm 0.11$ & 1487 & 1489 & $\beta$-Selinene & $0.41 \pm 0.03$ \\
\hline 948 & 946 & Camphene & $0.80 \pm 0.01$ & 1490 & 1490 & Phenylethyl 3-methylbutanoate & $0.09 \pm 0.01$ \\
\hline 971 & 969 & Sabinene & $1.96 \pm 0.00$ & 1493 & 1500 & Bicyclogermacrene & $1.03 \pm 0.00$ \\
\hline 976 & 974 & $\beta$-Pinene & $0.87 \pm 0.01$ & 1496 & 1500 & $\alpha$-Muurolene & $0.12 \pm 0.01$ \\
\hline 987 & 988 & Myrcene & $0.53 \pm 0.01$ & 1502 & 1509 & Lavandulyl 3-methylbutanoate & $0.76 \pm 0.01$ \\
\hline 1006 & 1002 & $\alpha$-Phellandrene & $28.30 \pm 0.16$ & 1511 & 1513 & $\gamma$-Cadinene & $0.21 \pm 0.01$ \\
\hline 1016 & 1014 & $\alpha$-Terpinene & $0.09 \pm 0.01$ & 1515 & 1518 & Bornyl 3-methylbutanoate & $0.39 \pm 0.01$ \\
\hline 1024 & 1020 & $p$-Cymene & $4.42 \pm 0.02$ & 1516 & 1522 & $\delta$-Cadinene & $0.36 \pm 0.01$ \\
\hline 1028 & 1024 & Limonene & $0.38 \pm 0.01$ & 1527 & 1529 & Kessane & $0.59 \pm 0.04$ \\
\hline 1030 & 1025 & $\beta$-Phellandrene & $0.06 \pm 0.02$ & 1535 & 1534 & Liguloxide & $0.84 \pm 0.01$ \\
\hline 1034 & 1032 & (Z)- $\beta$-Ocimene & $0.17 \pm 0.01$ & 1559 & 1561 & (E)-Nerolidol & $1.71 \pm 0.01$ \\
\hline 1044 & 1044 & (E)- $\beta$-Ocimene & $0.19 \pm 0.01$ & 1565 & 1565 & Thymyl 2-methylbutanoate & $0.69 \pm 0.01$ \\
\hline 1057 & 1054 & $\gamma$-Terpinene & $0.09 \pm 0.01$ & 1568 & 1570 & Neryl 2-methylbutanoate & $0.76 \pm 0.01$ \\
\hline 1069 & 1065 & cis-Sabinene hydrate & $0.08 \pm 0.01$ & 1575 & 1574 & Germacrene D- $4 \beta$-ol & $0.18 \pm 0.01$ \\
\hline 1084 & 1086 & Terpinolene & $0.07 \pm 0.01$ & 1581 & 1582 & Caryophyllene oxide & $0.21 \pm 0.02$ \\
\hline 1099 & 1095 & Linalool & $\operatorname{tr}^{c}$ & 1608 & 1613 & Copaborneol & $0.18 \pm 0.05$ \\
\hline 1101 & 1098 & trans-Sabinene hydrate & $\operatorname{tr}$ & 1641 & 1638 & $\tau$-Cadinol & $0.59 \pm 0.02$ \\
\hline 1141 & 1135 & trans-Pinocarveol & $0.08 \pm 0.02$ & 1654 & 1652 & $\alpha$-Cadinol & $0.81 \pm 0.02$ \\
\hline 1145 & 1140 & trans-Verbenol & $0.14 \pm 0.01$ & 1657 & 1658 & Selin-11-en- $4 \alpha$-ol & $0.15 \pm 0.01$ \\
\hline 1163 & 1165 & Lavandulol & $0.12 \pm 0.01$ & 1684 & 1685 & Germacra-4(15),5,10(14)-trien-1 $\alpha$-ol & $0.49 \pm 0.03$ \\
\hline 1172 & 1165 & Borneol & $0.11 \pm 0.01$ & 1693 & 1695 & 6-epi-Shyobunol & $0.20 \pm 0.02$ \\
\hline 1180 & 1174 & Terpinen-4-ol & $0.26 \pm 0.00$ & 2227 & $\mathrm{~d}$ & Kauran-16ß-ol & $3.48 \pm 0.01$ \\
\hline 1208 & 1204 & Verbenone & $0.06 \pm 0.01$ & 2243 & $\mathrm{~d}$ & Kauran-16 $\alpha$-ol & $0.17 \pm 0.02$ \\
\hline 1228 & 1232 & Thymol methyl ether & $2.89 \pm 0.01$ & & & Green leaf volatiles & 5.13 \\
\hline 1342 & 1345 & 7-epi-Silphiperfol-5-ene & $0.59 \pm 0.02$ & & & Monoterpene hydrocarbons & 57.78 \\
\hline 1351 & 1356 & Eugenol & $0.18 \pm 0.03$ & & & Oxygenated monoterpenoids & 6.34 \\
\hline 1367 & 1369 & Cyclosativene & $0.08 \pm 0.01$ & & & Sesquiterpene hydrocarbons & 18.92 \\
\hline 1367 & 1371 & Longicyclene & $\operatorname{tr}$ & & & Oxygenated sesquiterpenoids & 5.96 \\
\hline 1372 & 1377 & Silphiperol-6-ene & $0.06 \pm 0.00$ & & & Diterpenoids & 3.65 \\
\hline 1374 & 1374 & $\alpha$-Copaene & $0.12 \pm 0.01$ & & & Benzenoids & 0.45 \\
\hline 1380 & 1382 & Modheph-2-ene & $0.11 \pm 0.00$ & & & Others & 0.17 \\
\hline 1386 & 1387 & $\beta$-Cubebene & $0.06 \pm 0.01$ & & & Total Identified & 98.40 \\
\hline 1387 & 1389 & $\beta$-Elemene & $0.50 \pm 0.01$ & & & & \\
\hline
\end{tabular}

${ }^{\mathrm{a}} \mathrm{RI}=$ Retention index determined in reference to a homologous series of $n$-alkanes on a ZB-5ms column. ${ }^{\mathrm{b}} \mathrm{RI}$ values from the databases. ${ }^{c} \operatorname{tr}=$ "trace" $(<0.05 \%)$. ${ }^{\mathrm{d}}$ Assignment tentative; based on MS only.

\subsection{Rudbeckia laciniata L.}

Monoterpene hydrocarbons dominated the essential oil of $R$. laciniata (Table 6). The major components were limonene (58.07\%), $\alpha$-pinene $(10.18 \%), \beta$-pinene $(9.21 \%)$, and myrcene $(5.26 \%)$. While R. laciniata essential oil was rich in monoterpene hydrocarbons, the essential oils of $R$. fulgida and $R$. hirta were rich in sesquiterpene hydrocarbons [43]. The major components in $R$. fulgida essential oil were germacrene $\mathrm{D}(30.1 \%)$, $\delta$-cadinene $(17.8 \%), \beta$-caryophyllene $(10.0 \%)$, and $\gamma$-muurolene $(8.9 \%)$, along with $(E)$ - $\beta$-ocimene $(6.2 \%)$ and (2E)-hexenal (6.0\%). Similarly, the major components of $R$. hirta essential oil were germacrene $\mathrm{D}(23.6 \%), \delta$-cadinene $(16.2 \%), \beta$-caryophyllene $(4.7 \%), \gamma$-muurolene $(8.1 \%)$, as well as (E)- $\beta$-ocimene (15.2\%) and (2E)-hexenal (20.2\%) [43]. The leaf essential oil of Rudbeckia triloba, 
collected in Bucharest, Romania, was rich in monoterpene hydrocarbons, $\alpha$-pinene (46.0\%), sabinene (9.6\%), and $\beta$-phellandrene (24.6\%), along with germacrene D (6.1\%), but devoid of limonene [73]. Thus, there do not seem to be any consistent chemical markers for the Rudbeckia genus.

Table 6. Chemical composition of the essential oil of Rudbeckia laciniata L.

\begin{tabular}{|c|c|c|c|c|c|c|c|}
\hline $\mathrm{RI}^{\mathrm{a}}$ & $\mathbf{R I}^{b}$ & Compound & $\% \pm \mathrm{SD}$ & $\mathbf{R I}^{\mathrm{a}}$ & RI $^{b}$ & Compound & $\% \pm \mathrm{SD}$ \\
\hline 802 & 801 & Hexanal & $0.05 \pm 0.00$ & 1206 & 1204 & Verbenone & $0.10 \pm 0.03$ \\
\hline 810 & 796 & 2-Hexanol & $0.34 \pm 0.01$ & 1218 & 1215 & trans-Carveol & $0.25 \pm 0.06$ \\
\hline 922 & 921 & Tricyclene & $0.10 \pm 0.00$ & 1232 & 1226 & cis-Carveol & $0.07 \pm 0.02$ \\
\hline 924 & 924 & $\alpha$-Thujene & $0.10 \pm 0.01$ & 1243 & 1239 & Carvone & $0.49 \pm 0.02$ \\
\hline 932 & 932 & $\alpha$-Pinene & $10.18 \pm 0.06$ & 1283 & 1287 & Bornyl acetate & $2.68 \pm 0.02$ \\
\hline 948 & 946 & Camphene & $2.24 \pm 0.02$ & 1349 & 1350 & $\alpha$-Longipinene & $0.08 \pm 0.02$ \\
\hline 971 & 969 & Sabinene & $0.90 \pm 0.01$ & 1368 & 1369 & Cyclosativene & $0.06 \pm 0.02$ \\
\hline 977 & 974 & $\beta$-Pinene & $9.21 \pm 0.05$ & 1375 & 1374 & $\alpha$-Copaene & $0.16 \pm 0.01$ \\
\hline 988 & 988 & Myrcene & $5.26 \pm 0.02$ & 1391 & 1390 & Sativene & $0.05 \pm 0.01$ \\
\hline 1004 & 1003 & $p$-Mentha-1(7),8-diene & $0.07 \pm 0.01$ & 1417 & 1419 & $\beta$-Ylangene & $\operatorname{tr}$ \\
\hline 1024 & 1020 & $p$-Cymene & $0.11 \pm 0.01$ & 1418 & 1417 & $\beta$-Caryophyllene & $0.43 \pm 0.03$ \\
\hline 1029 & 1024 & Limonene & $58.07 \pm 0.47$ & 1428 & 1434 & $\gamma$-Elemene & $0.07 \pm 0.00$ \\
\hline 1030 & 1025 & $\beta$-Phellandrene & $0.74 \pm 0.08$ & 1431 & 1432 & trans- $\alpha$-Bergamotene & $0.19 \pm 0.02$ \\
\hline 1034 & 1032 & (Z)- $\beta$-Ocimene & $0.09 \pm 0.01$ & 1454 & 1452 & $\alpha$-Humulene & $0.12 \pm 0.01$ \\
\hline 1044 & 1044 & (E)- $\beta$-Ocimene & $1.07 \pm 0.03$ & 1473 & 1478 & $\gamma$-Muurolene & $0.05 \pm 0.01$ \\
\hline 1069 & 1067 & cis-Linalool oxide (furanoid) & $0.19 \pm 0.00$ & 1480 & 1484 & Germacrene D & $2.52 \pm 0.02$ \\
\hline 1085 & 1084 & trans-Linalool oxide (furanoid) & $0.05 \pm 0.01$ & 1482 & 1484 & $(Z, Z)-\alpha$-Farnesene & $0.05 \pm 0.01$ \\
\hline 1121 & 1119 & trans- $p$-Mentha-2,8-dien-1-ol & $0.37 \pm 0.01$ & 1494 & 1500 & Bicyclogermacrene & $0.06 \pm 0.01$ \\
\hline 1130 & 1131 & Limona ketone & $0.06 \pm 0.01$ & 1497 & 1500 & $\alpha$-Muurolene & $0.07 \pm 0.01$ \\
\hline 1132 & 1132 & cis-Limonene oxide & $0.20 \pm 0.00$ & 1514 & 1514 & Cubebol & $0.12 \pm 0.01$ \\
\hline 1136 & 1133 & cis-p-Mentha-2,8-dien-1-ol & $0.26 \pm 0.01$ & 1517 & 1522 & $\delta$-Cadinene & $0.15 \pm 0.01$ \\
\hline 1136 & 1137 & trans-Limonene oxide & $0.27 \pm 0.01$ & 1575 & 1574 & Germacra-1(10),5-dien- $4 \beta$-ol & $0.20 \pm 0.02$ \\
\hline 1138 & 1135 & Nopinone & $0.06 \pm 0.01$ & 1581 & 1582 & Caryophyllene oxide & $0.19 \pm 0.03$ \\
\hline 1140 & 1135 & trans-Pinocarveol & $0.19 \pm 0.03$ & 1591 & 1594 & Salvial-4(14)-en-1-one & $\operatorname{tr}$ \\
\hline 1145 & 1140 & trans-Verbenol & $0.07 \pm 0.01$ & 1601 & 1594 & Carotol & $0.18 \pm 0.01$ \\
\hline 1162 & 1160 & Pinocarvone & $0.12 \pm 0.00$ & 1620 & 1611 & Germacra-1(10),5-dien- $4 \alpha$-ol & $0.20 \pm 0.01$ \\
\hline 1171 & 1165 & Borneol & $0.11 \pm 0.02$ & & & Green leaf volatiles & 0.39 \\
\hline 1178 & 1179 & 2-Isopropenyl-5-methyl-4-hexenal & $0.08 \pm 0.01$ & & & Monoterpene hydrocarbons & 88.15 \\
\hline 1180 & 1174 & Terpinen-4-ol & $0.11 \pm 0.02$ & & & Oxygenated monoterpenoids & 6.18 \\
\hline 1187 & 1183 & Cryptone & $0.10 \pm 0.01$ & & & Sesquiterpene hydrocarbons & 4.06 \\
\hline 1195 & 1195 & Myrtenal & $0.23 \pm 0.02$ & & & Oxygenated sesquiterpenoids & 0.89 \\
\hline 1197 & 1200 & trans-Dihydrocarvone & $\operatorname{tr}^{c}$ & & & Total Identified & 99.67 \\
\hline 1199 & 1195 & cis-Piperitol & $0.11 \pm 0.07$ & & & & \\
\hline
\end{tabular}

\subsection{Silphium integrifolium Michx.}

The major components in the essential oil from the aerial parts of $S$. integrifolium were $\alpha$-pinene (58.59\%) and $\beta$-pinene (14.69\%), followed by myrcene (9.70\%; Table 7). Kowalski has extensively examined the essential oils of $S$. integrifolium as well as $S$. trifoliatum cultivated in Poland [74-78]. The leaf essential oil of S. integrifolium from Poland had $\alpha$-pinene (7.3-9.8\%), germacrene D (4.0-28.4\%), allo-aromadendrene (3.7-8.5\%), caryophyllene oxide (6.1-12.4\%), and silphiperfol-6-en-5-one (3.7-5.1\%) [74,75]; while the floral essential oil was made up of $\alpha$-pinene (13.4-14.0\%), camphene (5.3-5.7\%), trans-verbenol (5.2-6.3\%), bornyl acetate (6.5-7.0\%), and allo-aromadendrene (5.6-6.1\%) [74,77]. Thus, there are major qualitative and quantitative differences between the samples from Alabama and from Poland.

\subsection{Smallanthus uvedalia (L.) Mack.}

Monoterpene hydrocarbons dominated the essential oil of S. uvedalia (Table 8). $\alpha$-Pinene (62.56\%) was the major component, followed by limonene $(11.43 \%)$ and $\beta$-pinene $(6.00 \%)$. The chemical composition of this monoterpene-rich essential oil is very different from the compositions collected previously by us [45]. The previous samples, collected in February 2016, were dominated by $\beta$-caryophyllene $(24.5 \%$ and $16.5 \%$ ) and caryophyllene oxide (19.8\% and $14.2 \%)$. The sample of S. uvedalia in this present work was collected in September 2018. The differences in composition may be due to seasonal variation, genetic differences, or environmental stresses. Nevertheless, $\alpha$-pinene has dominated the essential oil compositions of other Smallanthus species. For example, $\alpha$-pinene was the major component in the essential oil of S. maculatus from Costa Rica (32.9\% $\alpha$-pinene), which was also rich in camphene (5.4\%), 
$\beta$-pinene (7.1\%), $\beta$-caryophyllene (10.7\%), germacrene D (13.7\%), and bicyclogermacrene (6.6\%) [79]. Likewise, the essential oil of $S$. quichensis from Costa Rica was also dominated by $\alpha$-pinene (35.5-64.5\%) with lesser concentrations of $\alpha$-phellandrene (0.1-9.0\%), p-cymene (0.1-11.5\%), limonene (2.1-5.8\%), $\beta$-phellandrene (up to 9.2\%), and 1,8-cineole (up to 9.7\%) [80]. In contrast, S. sonchifolia essential oil, grown in Sichuan, China, was made up of $\beta$-phellandrene (26.3\%), $\beta$-bourbonene $(10.2 \%)$, $\beta$-caryophyllene $(14.0 \%)$, and $\beta$-cubebene (17.6\%) [81].

Table 7. Chemical composition of the essential oil of Silphium integrifolium Michx.

\begin{tabular}{|c|c|c|c|c|c|c|c|}
\hline $\mathrm{RI}^{\mathrm{a}}$ & $\mathrm{RI}^{\mathrm{b}}$ & Compound & $\% \pm S D$ & $\mathbf{R I}^{\mathrm{a}}$ & $\mathbf{R I}^{b}$ & Compound & $\% \pm \mathrm{SD}$ \\
\hline 800 & 797 & (3Z)-Hexenal & $\operatorname{tr}^{\mathrm{c}}$ & 1387 & 1387 & $\beta$-Cubebene & $\operatorname{tr}$ \\
\hline 801 & 801 & Hexanal & $0.07 \pm 0.02$ & 1388 & 1389 & $\beta$-Elemene & $0.06 \pm 0.01$ \\
\hline 810 & 796 & 2-Hexanol & $\operatorname{tr}$ & 1417 & 1419 & $\beta$-Ylangene & $\operatorname{tr}$ \\
\hline 850 & 844 & (3E)-Hexenol & $0.27 \pm 0.04$ & 1429 & 1430 & $\beta$-Copaene & $\operatorname{tr}$ \\
\hline 922 & 921 & Tricyclene & $0.12 \pm 0.00$ & 1432 & 1432 & trans- $\alpha$-Bergamotene & $0.13 \pm 0.02$ \\
\hline 925 & 924 & $\alpha$-Thujene & $0.19 \pm 0.00$ & 1454 & 1452 & $\alpha$-Humulene & $1.07 \pm 0.02$ \\
\hline 949 & 946 & Camphene & $2.44 \pm 0.02$ & 1480 & 1484 & Germacrene D & $2.95 \pm 0.01$ \\
\hline 971 & 969 & Sabinene & $1.78 \pm 0.00$ & 1482 & 1484 & $(Z, Z)-\alpha$-Farnesene & $0.10 \pm 0.01$ \\
\hline 977 & 974 & $\beta$-Pinene & $14.69 \pm 0.07$ & 1488 & 1489 & $\beta$-Selinene & $0.15 \pm 0.01$ \\
\hline 988 & 988 & Myrcene & $9.70 \pm 0.02$ & 1491 & 1493 & trans-Muurola-4(14),5-diene & $\operatorname{tr}$ \\
\hline 1004 & 1003 & $p$-Mentha-1(7),8-diene & $\operatorname{tr}$ & 1494 & 1500 & Bicyclogermacrene & $0.08 \pm 0.00$ \\
\hline 1024 & 1020 & $p$-Cymene & $\operatorname{tr}$ & 1497 & 1500 & $\alpha$-Muurolene & $\operatorname{tr}$ \\
\hline 1085 & 1086 & Terpinolene & $\operatorname{tr}$ & 2019 & 2026 & $(E, E)$-Geranyl linalool & $0.06 \pm 0.01$ \\
\hline 1099 & 1099 & $\alpha$-Pinene oxide & $0.10 \pm 0.01$ & 2228 & 2237 & $7 \alpha$-Hydroxymanool & $0.16 \pm 0.02$ \\
\hline 1112 & 1113 & (E)-4,8-Dimethylnona-1,3,7-triene & $\operatorname{tr}$ & 2300 & 2300 & Tricosane & $\operatorname{tr}$ \\
\hline 1126 & 1122 & $\alpha$-Campholenal & $\operatorname{tr}$ & 2500 & 2500 & Pentacosane & $0.16 \pm 0.01$ \\
\hline 1140 & 1135 & trans-Pinocarveol & $\operatorname{tr}$ & 2700 & 2700 & Heptacosane & $0.16 \pm 0.02$ \\
\hline 1145 & 1140 & trans-Verbenol & $0.11 \pm 0.02$ & & & Green leaf volatiles & 0.66 \\
\hline 1162 & 1160 & Pinocarvone & $\operatorname{tr}$ & & & Monoterpene hydrocarbons & 90.09 \\
\hline 1180 & 1174 & Terpinen-4-ol & $\operatorname{tr}$ & & & Oxygenated monoterpenoids & 0.38 \\
\hline 1195 & 1195 & Myrtenal & $0.06 \pm 0.01$ & & & Sesquiterpene hydrocarbons & 7.37 \\
\hline 1206 & 1204 & Verbenone & $0.10 \pm 0.02$ & & & Oxygenated sesquiterpenoids & 1.09 \\
\hline 1368 & 1369 & Cyclosativene & $0.06 \pm 0.00$ & & & Others & 0.32 \\
\hline 1375 & 1374 & $\alpha$-Copaene & $0.08 \pm 0.01$ & & & Total Identified & 99.90 \\
\hline
\end{tabular}

${ }^{\mathrm{a}} \mathrm{RI}=$ Retention index determined in reference to a homologous series of $n$-alkanes on a ZB-5ms column. ${ }^{\mathrm{b}} \mathrm{RI}$ values from the databases. ${ }^{\mathrm{c}} \operatorname{tr}=$ "trace" $(<0.05 \%)$.

Table 8. Chemical composition of the essential oil of Smallanthus uvedalia (L.) Mack.

\begin{tabular}{|c|c|c|c|c|c|c|c|}
\hline $\mathrm{RI}^{\mathrm{a}}$ & RI $^{b}$ & Compound & $\% \pm \mathrm{SD}$ & $\mathrm{RI}^{\mathrm{a}}$ & RI $^{b}$ & Compound & $\% \pm \mathrm{SD}$ \\
\hline 795 & 801 & 2-Methylhept-2-ene & $0.10 \pm 0.00$ & 1207 & 1204 & Verbenone & $0.09 \pm 0.01$ \\
\hline 801 & 801 & Hexanal & $0.86 \pm 0.16$ & 1346 & 1345 & $\alpha$-Cubebene & $0.15 \pm 0.04$ \\
\hline 850 & 846 & (2E)-Hexenal & $1.40 \pm 0.09$ & 1382 & 1387 & $\beta$-Bourbonene & $0.15 \pm 0.02$ \\
\hline 922 & 921 & Tricyclene & $0.08 \pm 0.00$ & 1454 & 1452 & $\alpha$-Humulene & $0.36 \pm 0.02$ \\
\hline 924 & 924 & $\alpha$-Thujene & $1.28 \pm 0.02$ & 1473 & 1478 & $\gamma$-Muurolene & $0.56 \pm 0.12$ \\
\hline 932 & 932 & $\alpha$-Pinene & $62.56 \pm 0.79$ & 1512 & 1513 & $\gamma$-Cadinene & $0.29 \pm 0.09$ \\
\hline 948 & 946 & Camphene & $1.35 \pm 0.01$ & 1517 & 1522 & $\delta$-Cadinene & $0.63 \pm 0.03$ \\
\hline 971 & 969 & Sabinene & $0.16 \pm 0.03$ & 1536 & 1537 & $\alpha$-Cadinene & $0.22 \pm 0.04$ \\
\hline 977 & 974 & $\beta$-Pinene & $6.00 \pm 0.09$ & 1576 & 1577 & Spathulenol & $0.58 \pm 0.11$ \\
\hline 988 & 988 & Myrcene & $2.43 \pm 0.07$ & 1581 & 1582 & Caryophyllene oxide & $1.37 \pm 0.02$ \\
\hline 1024 & 1020 & $p$-Cymene & $0.15 \pm 0.01$ & & & Green leaf volatiles & 2.48 \\
\hline 1028 & 1024 & Limonene & $11.43 \pm 0.11$ & & & Monoterpene hydrocarbons & 88.28 \\
\hline 1030 & 1025 & $\beta$-Phellandrene & $0.70 \pm 0.10$ & & & Oxygenated monoterpenoids & 0.74 \\
\hline 1044 & 1044 & (E)- $\beta$-Ocimene & $1.87 \pm 0.09$ & & & Sesquiterpene hydrocarbons & 6.16 \\
\hline 1057 & 1054 & $\gamma$-Terpinene & $0.26 \pm 0.01$ & & & Oxygenated sesquiterpenoids & 1.95 \\
\hline 1126 & 1122 & $\alpha$-Campholenal & $0.29 \pm 0.01$ & & & Others & 0.10 \\
\hline 1140 & 1135 & trans-Pinocarveol & $0.23 \pm 0.04$ & & & Total Identified & 99.71 \\
\hline 1145 & 1140 & trans-Verbenol & $0.14 \pm 0.05$ & & & & \\
\hline
\end{tabular}

${ }^{\mathrm{a}} \mathrm{RI}=$ Retention index determined in reference to a homologous series of $n$-alkanes on a ZB- $5 \mathrm{~ms}$ column. ${ }^{\mathrm{b}} \mathrm{RI}$ values from the databases. 


\subsection{Solidago altissima L. (syn. Solidago canadensis L.)}

The major components in the essential oil from the aerial parts of S. altissima (syn. S. canadensis) from Alabama were $\alpha$-pinene (13.91\%), sabinene (14.25\%), myrcene $(20.29 \%)$, bornyl acetate $(14.44 \%)$, and germacrene D (10.67\%; Table 9). Previous examinations of $S$. canadensis essential oils have shown germacrene $\mathrm{D}$ to be one of the most abundant components (Table 10). However, Weyerstahl and co-workers [82] found curlone $(23.5 \%)$ to be a major component of S. canadensis from Poland, Schmidt and co-workers [83] found cyclocolorenone (38\%) to be a major component in S. canadensis from northern Germany, and Kasali and co-workers [84] found 6-epi- $\beta$-cubebene to be a major component $(20.5 \%)$ in S. canadensis essential oil from Poland. Interestingly, none of these compounds was detected in the sample of $S$. altissima essential oil from Alabama.

Table 9. Chemical composition of the essential oil of Solidago altissima L.

\begin{tabular}{|c|c|c|c|c|c|c|c|}
\hline $\mathrm{RI}^{\mathrm{a}}$ & RI $^{b}$ & Compound & $\% \pm \mathrm{SD}$ & $\mathrm{RI}^{\mathrm{a}}$ & $\mathrm{RI}^{\mathrm{b}}$ & Compound & $\% \pm \mathrm{SD}$ \\
\hline 802 & 801 & Hexanal & $0.17 \pm 0.01$ & 1382 & 1387 & $\beta$-Bourbonene & $\operatorname{tr}$ \\
\hline 850 & 846 & (2E)-Hexenal & $1.21 \pm 0.03$ & 1386 & 1387 & $\beta$-Cubebene & $0.10 \pm 0.01$ \\
\hline 921 & 921 & Tricyclene & $0.06 \pm 0.00$ & 1387 & 1389 & $\beta$-Elemene & $0.18 \pm 0.00$ \\
\hline 931 & 932 & $\alpha$-Pinene & $13.91 \pm 0.04$ & 1418 & 1417 & $\beta$-Caryophyllene & $0.50 \pm 0.03$ \\
\hline 948 & 946 & Camphene & $2.41 \pm 0.01$ & 1428 & 1430 & $\beta$-Copaene & $0.14 \pm 0.01$ \\
\hline 971 & 969 & Sabinene & $14.25 \pm 0.03$ & 1454 & 1452 & $\alpha$-Humulene & $0.17 \pm 0.00$ \\
\hline 1005 & 1004 & (3Z)-Hexenyl acetate & $\operatorname{tr}^{c}$ & 1479 & 1484 & Germacrene D & $10.67 \pm 0.03$ \\
\hline 1006 & 1002 & $\alpha$-Phellandrene & $2.84 \pm 0.02$ & 1487 & 1489 & $\beta$-Selinene & $\operatorname{tr}$ \\
\hline 1016 & 1014 & $\alpha$-Terpinene & $0.10 \pm 0.00$ & 1490 & 1495 & $\gamma$-Amorphene & $0.59 \pm 0.01$ \\
\hline 1024 & 1020 & $p$-Cymene & $2.26 \pm 0.00$ & 1494 & 1500 & Bicyclogermacrene & $0.18 \pm 0.00$ \\
\hline 1028 & 1024 & Limonene & $1.27 \pm 0.01$ & 1496 & 1500 & $\alpha$-Muurolene & $0.14 \pm 0.01$ \\
\hline 1030 & 1025 & $\beta$-Phellandrene & $0.35 \pm 0.01$ & 1511 & 1513 & $\gamma$-Cadinene & $0.30 \pm 0.01$ \\
\hline 1090 & 1090 & 6,7-Epoxymyrcene & $0.05 \pm 0.00$ & 1575 & 1574 & Germacra- $1(10), 5$-dien- $4 \beta$-ol & $0.14 \pm 0.01$ \\
\hline 1099 & 1099 & $\alpha$-Pinene oxide & $\operatorname{tr}$ & 1581 & 1582 & Caryophyllene oxide & $0.06 \pm 0.01$ \\
\hline 1101 & 1098 & trans-Sabinene hydrate & $0.15 \pm 0.00$ & 1591 & 1594 & Salvial-4(14)-en-1-one & $0.06 \pm 0.01$ \\
\hline 1105 & 1100 & Nonanal & $\operatorname{tr}$ & 1619 & 1611 & Germacra- $1(10), 5$-dien- $4 \alpha$-ol & $0.12 \pm 0.01$ \\
\hline 1112 & 1113 & (E)-4,8-Dimethylnona-1,3,7-triene & $0.11 \pm 0.03$ & 1627 & 1629 & iso-Spathulenol & $0.20 \pm 0.01$ \\
\hline 1124 & 1124 & cis- $p$-Menth-2-en-1-ol & $0.05 \pm 0.00$ & 1641 & 1638 & $\tau$-Cadinol & $0.09 \pm 0.02$ \\
\hline 1180 & 1174 & Terpinen-4-ol & $0.73 \pm 0.01$ & 1643 & 1640 & $\tau$-Murrolol & $0.14 \pm 0.01$ \\
\hline 1195 & 1186 & $\alpha$-Terpineol & $0.06 \pm 0.01$ & 1645 & 1644 & $\alpha$-Muurolol (= $\delta$-Cadinol) & $0.10 \pm 0.01$ \\
\hline 1203 & 1202 & cis-Sabinol & $0.50 \pm 0.01$ & 1654 & 1652 & $\alpha$-Cadinol & $0.41 \pm 0.03$ \\
\hline 1219 & 1219 & cis-Sabinene hydrate acetate & $0.15 \pm 0.01$ & & & Green leaf volatiles & 1.38 \\
\hline 1283 & 1287 & Bornyl acetate & $14.44 \pm 0.02$ & & & Monoterpene hydrocarbons & 64.26 \\
\hline 1333 & 1335 & $\delta$-Elemene & $0.05 \pm 0.00$ & & & Oxygenated monoterpenoids & 16.37 \\
\hline 1345 & 1345 & $\alpha$-Cubebene & $0.12 \pm 0.00$ & & & Sesquiterpene hydrocarbons & 14.90 \\
\hline
\end{tabular}

${ }^{\mathrm{a}} \mathrm{RI}=$ Retention index determined in reference to a homologous series of $n$-alkanes on a ZB-5ms column. ${ }^{\mathrm{b}} \mathrm{RI}$ values from the databases. ${ }^{\mathrm{c}} \operatorname{tr}=$ "trace" $(<0.05 \%)$.

Table 10. Comparison of the major components in Solidago altissima (syn. S. canadensis) essential oils.

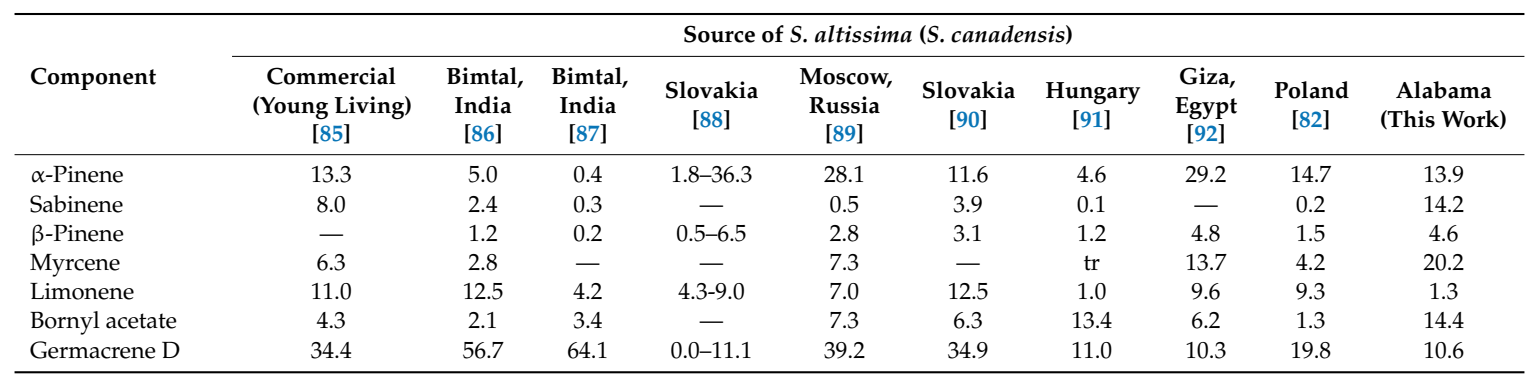




\subsection{Xanthium strumarium L.}

The major volatile components from the aerial parts of X. strumarium were limonene (48.23\%), myrcene $(14.31 \%)$, germacrene D $(13.92 \%)$, (2E)-hexenal $(5.79 \%)$, and sabinene $(4.89 \%$; Table 11$)$. The compositions of Xanthium strumarium essential oils from the Middle East have been reported, including Iran [93,94] and Pakistan [95]. The leaf essential oil from Khoramabad, Iran, was composed largely of limonene (24.7\%), borneol (10.6\%), bornyl acetate (5.9\%), and $\beta$-cubebene (6.3\%) [93]. The leaf essential oil from Zabol, Iran, was qualitatively similar, limonene (20.3\%), borneol (11.6\%), bornyl acetate $(4.5 \%)$, and $\beta$-cubebene $(3.8 \%)$, but also contained a large concentration of $c$ is- $\beta$-guaiene $(34.2 \%)$, which was not observed in any other X. strumarium essential oils [94]. The leaf essential oil of X. strumarium from Lahore, Pakistan, contained limonene (5.7\%), $\beta$-caryophyllene (17.5\%), spathulenol (6.1\%), and $\alpha$-cadinol (6.7\%) as major components [95]. The differences in chemical compositions may be related to different genetic factors as well as geographical location; Tropicos ${ }^{\circledR}$ currently lists 13 subordinate taxa for X. strumarium [14].

Table 11. Chemical composition of the essential oil of Xanthium strumarium L.

\begin{tabular}{|c|c|c|c|c|c|c|c|}
\hline $\mathrm{RI}^{\mathrm{a}}$ & $\mathbf{R I}^{\mathbf{b}}$ & Compound & $\% \pm \mathrm{SD}$ & RI $^{a}$ & RI $^{b}$ & Compound & $\% \pm \mathrm{SD}$ \\
\hline 793 & 788 & 1-Octene & $0.09 \pm 0.01$ & 1417 & 1417 & $\beta$-Caryophyllene & $0.93 \pm 0.04$ \\
\hline 801 & 797 & (3Z)-Hexenal & $0.11 \pm 0.01$ & 1428 & 1430 & $\beta$-Copaene & $0.06 \pm 0.01$ \\
\hline 802 & 801 & Hexanal & $0.75 \pm 0.07$ & 1454 & 1452 & $\alpha$-Humulene & $0.49 \pm 0.03$ \\
\hline 859 & 846 & (2E)-Hexenal & $5.79 \pm 0.03$ & 1479 & 1484 & Germacrene D & $13.92 \pm 0.05$ \\
\hline 865 & 863 & 1-Hexanol & $0.12 \pm 0.01$ & 1487 & 1489 & $\beta$-Selinene & $0.11 \pm 0.01$ \\
\hline 921 & 921 & Tricyclene & $0.05 \pm 0.01$ & 1493 & 1500 & Bicyclogermacrene & $0.29 \pm 0.01$ \\
\hline 924 & 924 & $\alpha$-Thujene & $0.08 \pm 0.03$ & 1496 & 1500 & $\alpha$-Muurolene & $0.13 \pm 0.01$ \\
\hline 931 & 932 & $\alpha$-Pinene & $0.80 \pm 0.01$ & 1511 & 1513 & $\gamma$-Cadinene & $0.19 \pm 0.01$ \\
\hline 948 & 946 & Camphene & $0.95 \pm 0.02$ & 1516 & 1522 & $\delta$-Cadinene & $0.27 \pm 0.03$ \\
\hline 971 & 969 & Sabinene & $4.89 \pm 0.02$ & 1575 & 1547 & Germacra- $1(10), 5$-dien- $4 \beta$-ol & $0.21 \pm 0.01$ \\
\hline 976 & 974 & $\beta$-Pinene & $0.30 \pm 0.01$ & 1581 & 1582 & Caryophyllene oxide & $0.23 \pm 0.01$ \\
\hline 978 & 974 & 1-Octen-3-ol & $0.20 \pm 0.02$ & 1641 & 1638 & $\tau$-Cadinol & $0.31 \pm 0.02$ \\
\hline 987 & 988 & Myrcene & $14.31 \pm 0.04$ & 1643 & 1640 & $\tau$-Muurolol & $0.23 \pm 0.02$ \\
\hline 1004 & 1003 & $p$-Mentha-1(7),8-diene & $0.05 \pm 0.01$ & 1654 & 1652 & $\alpha$-Cadinol & $0.59 \pm 0.05$ \\
\hline 1016 & 1014 & $\alpha$-Terpinene & $0.06 \pm 0.01$ & 1663 & 1668 & ar-Turmerone & $0.10 \pm 0.01$ \\
\hline 1028 & 1024 & Limonene & $48.23 \pm 0.22$ & 1693 & 1688 & Shyobunol & $0.27 \pm 0.03$ \\
\hline 1030 & 1025 & $\beta$-Phellandrene & $0.90 \pm 0.03$ & 1932 & 1931 & Beyerene & $0.64 \pm 0.02$ \\
\hline 1044 & 1044 & (E)- $\beta$-Ocimene & $0.10 \pm 0.02$ & 2105 & 2106 & (E)-Phytol & $0.25 \pm 0.03$ \\
\hline 1057 & 1054 & $\gamma$-Terpinene & $0.16 \pm 0.01$ & & & Green leaf volatiles & 6.77 \\
\hline 1069 & 1067 & cis-Linalool oxide (furanoid) & $0.06 \pm 0.01$ & & & Monoterpene hydrocarbons & 70.87 \\
\hline 1099 & 1095 & Linalool & $1.16 \pm 0.01$ & & & Oxygenated monoterpenoids & 2.22 \\
\hline 1180 & 1174 & Terpinen-4-ol & $0.39 \pm 0.01$ & & & Sesquiterpene hydrocarbons & 16.58 \\
\hline 1219 & 1217 & $\beta$-Cyclocitral & $0.11 \pm 0.02$ & & & Oxygenated sesquiterpenoids & 1.95 \\
\hline 1283 & 1287 & Bornyl acetate & $0.56 \pm 0.01$ & & & Diterpenoids & 0.89 \\
\hline 1351 & 1356 & Eugenol & $0.28 \pm 0.03$ & & & Benzenoids & 0.28 \\
\hline 1386 & 1387 & $\beta$-Cubebene & $0.10 \pm 0.02$ & & & Others & 0.29 \\
\hline 1416 & 1419 & $\beta$-Ylangene & $0.08 \pm 0.03$ & & & Total Identified & 99.85 \\
\hline
\end{tabular}

\subsection{Antifungal Screening}

Depending on material available, the essential oils were screened for antifungal activity against the opportunistic fungal pathogens Aspergillus niger, Candida albicans, and Cryptococcus neoformans using the microbroth dilution technique (Table 12). The essential oil of E. serotinum showed promising antifungal activity against $C$. neoformans with a minimum inhibitory concentration (MIC) value of $78 \mu \mathrm{g} / \mathrm{mL}$. The high concentration of cyclocolorenone in E. serotinum is likely responsible for the observed antifungal activity of this essential oil. Cyclocolorenone had been previously reported to show antifungal activity against Curvularia lunata, Chaetomium cochliodes, and Chaetomium spinusum [96]. Germacrene D may also contribute to the antifungal activity of E. serotinum essential oil as well as essential oils of E. macrophylla, P. canadensis, and R. laciniata. Germacrene D has shown antifungal activity against Aspergillus niger with MIC of $39 \mu \mathrm{g} / \mathrm{mL}$ [97]. 
Table 12. Major components and antifungal activities of Asteraceae essential oils.

\begin{tabular}{|c|c|c|c|c|}
\hline \multirow{2}{*}{ Plant Species } & \multirow{2}{*}{ Major Components (>5\%) in the Essential Oil } & \multicolumn{3}{|c|}{ Antifungal Activity, $\mathrm{MIC}, \mu \mathrm{g} / \mathrm{mL}^{\mathrm{a}}$} \\
\hline & & $\begin{array}{l}\text { Aspergillus } \\
\text { niger }\end{array}$ & $\begin{array}{l}\text { Candida } \\
\text { albicans }\end{array}$ & $\begin{array}{l}\text { Cryptococcus } \\
\text { neoformans }\end{array}$ \\
\hline Eupatorium serotinum Michx. & $\begin{array}{l}\text { germacrene D }(6.6 \%) \text {, palustrol }(5.4 \%) \text {, } \\
\text { cyclocolorenone }(23.5 \%)\end{array}$ & 313 & 625 & 78 \\
\hline Eurybia macrophylla (L.) Cass. & $\begin{array}{l}\beta \text {-pinene }(8.5 \%) \text {, limonene }(28.6 \%) \text {, terpinolene } \\
(5.3 \%) \text {, germacrene D }(19.7 \%) \text {, germacrene B }(7.0 \%)\end{array}$ & 625 & 625 & 156 \\
\hline $\begin{array}{l}\text { Eutrochium purpureum (L.) } \\
\text { E.E. Lamont }\end{array}$ & $\begin{array}{l}\text { hexanal }(6.8 \%),(2 E) \text {-hexenal }(59.7 \%) \text {, methyl } \\
\text { salicylate }(10.4 \%) \text {, eugenol }(11.8 \%)\end{array}$ & 625 & 625 & 625 \\
\hline Polymnia canadensis L. & $\begin{array}{l}\alpha \text {-pinene }(19.6 \%), \alpha \text {-phellandrene }(28.2 \%), \\
\text { germacrene D }(11.4 \%)\end{array}$ & 625 & 625 & 156 \\
\hline Rudbeckia laciniata $\mathrm{L}$. & $\begin{array}{l}\alpha \text {-pinene }(10.2 \%), \beta \text {-pinene }(9.2 \%), \text { myrcene }(5.3 \%), \\
\text { limonene }(58.9 \%)\end{array}$ & 625 & 1250 & 156 \\
\hline Silphium integrifolium Michx. & $\alpha$-pinene $(58.5 \%), \beta$-pinene $(14.7 \%)$, myrcene $(9.7 \%)$ & n.t. ${ }^{b}$ & n.t. & n.t. \\
\hline $\begin{array}{l}\text { Smallanthus uvedalia (L.) } \\
\text { Mack. }\end{array}$ & $\alpha$-pinene $(62.3 \%), \beta$-pinene $(6.0 \%)$, limonene $(11.3 \%)$ & n.t. & n.t. & n.t. \\
\hline Solidago altissima $\mathrm{L}$. & $\begin{array}{l}\alpha \text {-pinene }(13.9 \%) \text {, sabinene }(14.2 \%) \text {, myrcene }(20.2 \%) \text {, } \\
\text { bornyl acetate }(14.4 \%) \text {, germacrene D }(10.6 \%)\end{array}$ & 625 & 1250 & 313 \\
\hline Xanthium strumarium $\mathrm{L}$. & $\begin{array}{l}\text { (2E)-hexenal }(5.8 \%) \text {, myrcene }(14.3 \%) \text {, limonene } \\
(48.0 \%) \text {, germacrene D }(13.9 \%)\end{array}$ & 625 & 1250 & n.t. \\
\hline
\end{tabular}

${ }^{a}$ Each minimum inhibitory concentration (MIC) determination was carried out in triplicate. ${ }^{b}$ n.t. $=$ not tested due to limited availability of the essential oil.

The modest antifungal activity of E. purpureum is somewhat surprising. The major components were hexanal, (2E)-hexenal, methyl salicylate, and eugenol. Hexanal [98] and (2E)-hexenal $[99,100]$ are both known to be antifungal to plant pathogenic fungi. Methyl salicylate is only weakly antifungal against $A$. niger, $C$. albicans, or $C$. neoformans, but eugenol is somewhat active (see Table 13). Monoterpene hydrocarbons such as $\alpha$-pinene, $\beta$-pinene, limonene, or myrcene show only weak antifungal activity (Table 13) and are not expected to contribute to the antifungal activities of the essential oils unless there are synergistic effects of these components (see, for example [101,102]). The mechanisms of antifungal activity of essential oils are poorly understood. However, it has been suggested that essential oils and their components, being lipophilic, can disrupt the membranes of fungi causing membrane permeability [103].

Table 13. Antifungal activities (MIC, $\mu \mathrm{g} / \mathrm{mL}$ ) of essential oil components.

\begin{tabular}{cccc}
\hline Compound & Aspergillus niger & Candida albicans & Cryptococcus neoformans \\
\hline$\alpha$-Pinene & 1250 & 625 & 313 \\
$\beta$-Pinene & 625 & 1250 & 625 \\
Limonene & 625 & 1250 & 625 \\
Myrcene & 625 & 625 & 625 \\
Methyl salicylate & 625 & 625 & 625 \\
Eugenol & 78 & 313 & 156 \\
Bornyl acetate & 625 & 625 & 625 \\
\hline
\end{tabular}

\section{Materials and Methods}

\subsection{Plant Material}

Aerial parts of each plant were collected from various sites in north Alabama (Table 14). Plants were identified by S.K. Lawson and voucher specimens were deposited in the University of Alabama in Huntsville herbarium (HALA). The fresh plant material (aerial parts) were chopped and hydrodistilled using a Likens-Nickerson apparatus with continuous extraction with $\mathrm{CH}_{2} \mathrm{Cl}_{2}$ for three hours. The solvent was evaporated to give pale yellow essential oils (Table 14). 
Table 14. Plant collection sites and essential oil yields of Asteraceae from north Alabama.

\begin{tabular}{|c|c|c|c|c|}
\hline Plant & Collection Site, Date & $\begin{array}{l}\text { Voucher } \\
\text { Number }\end{array}$ & $\begin{array}{l}\text { Mass of Aerial } \\
\text { Parts (g) }\end{array}$ & $\begin{array}{l}\text { Yield of Essential } \\
\text { Oil (mg) }\end{array}$ \\
\hline $\begin{array}{l}\text { Eupatorium serotinum } \\
\text { Michx. }\end{array}$ & $\begin{array}{c}34^{\circ} 38^{\prime} 29^{\prime \prime} \mathrm{N}, 86^{\circ} 24^{\prime} 39^{\prime \prime} \mathrm{W} \\
\text { elev. } 199 \mathrm{~m} \\
13 \text { September } 2018\end{array}$ & 233754 & 49.09 & $6.4(0.013 \%)$ \\
\hline $\begin{array}{l}\text { Eurybia macrophylla (L.) } \\
\text { Cass. }\end{array}$ & $\begin{array}{c}34^{\circ} 39^{\prime} 25^{\prime \prime} \mathrm{N}, 86^{\circ} 24^{\prime} 45^{\prime \prime} \mathrm{W} \\
\text { elev. } 241 \mathrm{~m} \\
15 \text { September } 2018\end{array}$ & 233117 & 56.56 & $10.6(0.019 \%)$ \\
\hline $\begin{array}{l}\text { Eutrochium purpureum } \\
\text { (L.) E.E. Lamont }\end{array}$ & $\begin{array}{c}34^{\circ} 38^{\prime} 40^{\prime \prime} \mathrm{N}, 86^{\circ} 27^{\prime} 22^{\prime \prime} \mathrm{W}, \\
\text { elev. } 180 \mathrm{~m} \\
12 \text { August } 2018\end{array}$ & 091843 & 63.44 & $12.3(0.019 \%)$ \\
\hline Polymnia canadensis L. & $\begin{array}{c}34^{\circ} 38^{\prime} 29^{\prime \prime} \mathrm{N}, 86^{\circ} 24^{\prime} 39^{\prime \prime} \mathrm{W} \\
\text { elev. } 199 \mathrm{~m} \\
\text { 21 July } 2018\end{array}$ & 184700 & 52.89 & $39.1(0.074 \%)$ \\
\hline Rudbeckia laciniata $\mathrm{L}$. & $\begin{array}{c}34^{\circ} 42^{\prime} 42^{\prime \prime} \mathrm{N}, 86^{\circ} 32^{\prime} 38^{\prime \prime} \mathrm{W}, \\
\text { elev. } 345 \mathrm{~m} \\
13 \text { September } 2018\end{array}$ & 004426 & 54.53 & $6.0(0.011 \%)$ \\
\hline $\begin{array}{l}\text { Silphium integrifolium } \\
\text { Michx. }\end{array}$ & $\begin{array}{c}34^{\circ} 42^{\prime} 42^{\prime \prime} \mathrm{N}, 86^{\circ} 32^{\prime} 38^{\prime \prime} \mathrm{W}, \\
\text { elev. } 345 \mathrm{~m} \\
15 \text { September } 2018\end{array}$ & 004152 & 15.02 & $6.4(0.043 \%)$ \\
\hline $\begin{array}{l}\text { Smallanthus uvedalia (L.) } \\
\text { Mack. }\end{array}$ & $\begin{array}{c}34^{\circ} 42^{\prime} 42^{\prime \prime} \mathrm{N}, 86^{\circ} 32^{\prime} 38^{\prime \prime} \mathrm{W}, \\
\text { elev. } 345 \mathrm{~m} \\
15 \text { September } 2018\end{array}$ & 000714 & 56.21 & $5.9(0.010 \%)$ \\
\hline Solidago altissima $\mathrm{L}$. & $\begin{array}{c}34^{\circ} 38^{\prime} 40^{\prime \prime} \mathrm{N}, 86^{\circ} 27^{\prime} 22^{\prime \prime} \mathrm{W}, \\
\text { elev. } 180 \mathrm{~m} \\
12 \text { August } 2018\end{array}$ & 001425 & 54.41 & $44.9(0.083 \%)$ \\
\hline Xanthium strumarium L. & $\begin{array}{c}34^{\circ} 38^{\prime} 49^{\prime \prime} \mathrm{N}, 86^{\circ} 24^{\prime} 38^{\prime \prime} \mathrm{W}, \\
\text { elev. } 200 \mathrm{~m} \\
15 \text { September } 2018\end{array}$ & 224724 & 69.69 & $7.0(0.010 \%)$ \\
\hline
\end{tabular}

\subsection{Gas Chromatography-Mass Spectrometry}

The Asteraceae essential oils were analyzed by GC-MS using a Shimadzu GC-MS-QP2010 Ultra fitted with a Phenomenex ZB-5ms column as previously described [104]. Identification of the essential oil components was determined by comparison of their retention indices, determined with respect to a homologous series of $n$-alkanes and their mass spectral fragmentation patters with those from available databases (Adams [105], NIST17 [106], and FFNSC 3 [107]) or in our in-house library [108].

\subsection{Gas Chromatography-Flame Ionization Detection}

Quantification of the essential oils was determined by GC-FID using a Shimadzu GC 2010 instrument fitted with a ZB-5 column [104], using the same parameters that were used for the GC-MS. The concentrations (average of three measurements \pm standard deviations) are based on peak integration without standardization.

\subsection{Antifungal Screening}

The essential oils were screened for antifungal activity against Aspergillus niger (ATCC 16888), Candida albicans (ATCC 18804), and Cryptococcus neoformans (ATCC 24607) using the microbroth dilution method as previously described [109]. Amphotericin B was used as the positive control and RPMI medium was used as the negative control. The antifungal assays were carried out in triplicate.

\section{Conclusions}

There is much intraspecific variation in essential oil compositions of these members of the Asteraceae. Much of the variation can be attributed to geographical location or seasonal variation. 
Eupatorium serotinum essential oil showed notable antifungal activity against Cryptococcus neoformans. However, the yield of this essential oil $(0.013 \%)$ is too low to be considered as pharmacologically useful. If suitable sources of the major component cyclocolorenone can be identified, then this compound may serve as important antifungal template for further elaboration.

Author Contributions: Conceptualization, S.K.L. and W.N.S.; methodology, W.N.S., R.L.M., and P.S.; software, P.S.; validation, W.N.S.; formal analysis, W.N.S.; investigation, S.K.L., L.G.S., C.N.P., and P.S.; resources, R.L.M., P.S., and W.N.S.; data curation, W.N.S.; writing—original draft preparation, W.N.S.; writing—review and editing, S.K.L., L.G.S., C.N.P., R.L.M., P.S., and W.N.S.; supervision, W.N.S. and R.L.M.; project administration, W.N.S. All authors have read and agreed to the published version of the manuscript.

Funding: This research received no external funding.

Acknowledgments: P.S. and W.N.S. participated in the project as part of the activities of the Aromatic Plant Research Center (APRC, https://aromaticplant.org/).

Conflicts of Interest: The authors declare no conflict of interest.

\section{References}

1. NCCIH Ayurvedic Medicine: In Depth. Available online: https://nccih.nih.gov/health/ayurveda/introduction. htm (accessed on 17 July 2019).

2. Shang, A.; Huwiler, K.; Nartey, L.; Jüni, P.; Egger, M. Placebo-controlled trials of Chinese herbal medicine and conventional medicine-Comparative study. Int. J. Epidemiol. 2007, 36, 1086-1092. [CrossRef]

3. Avicenna. Canon of Medicine; Kazi Publications: Chicago, IL, USA, 2015; ISBN 978-1567442243.

4. Setzer, W.N. The phytochemistry of Cherokee aromatic medicinal plants. Medicines 2018, 5, 121. [CrossRef]

5. Kartesz, J.T. BONAP's North American Plant Atlas. Available online: http://bonap.net/MapGallery/County/ Conocliniumcoelestinum.png (accessed on 7 August 2019).

6. Moerman, D.E. Native American Ethnobotany; Timber Press, Inc.: Portland, OR, USA, 1998.

7. Herz, W.; de Groote, R.; Murari, R.; Kumar, N.; Blount, J.F. Sesquiterpene lactones of Eupatorium serotinum. J. Org. Chem. 1979, 44, 2784-2788. [CrossRef]

8. Bohlmann, F.; Zdero, C.; King, R.M.; Robinson, H. Further germacranolides from Eupatorium serotinum. Planta Med. 1985, 51, 76-77. [CrossRef]

9. Kartesz, J.T. BONAP's North American Plant Atlas. Available online: http://bonap.net/Napa/TaxonMaps/ Genus/County/Eurybia (accessed on 17 July 2019).

10. Kartesz, J.T. BONAP's North American Plant Atlas. Available online: http://bonap.net/Napa/TaxonMaps/ Genus/County/Eutrochium (accessed on 17 July 2019).

11. Kartesz, J.T. BONAP's North American Plant Atlas. Available online: http://bonap.net/Napa/TaxonMaps/ Genus/County/Polymnia (accessed on 17 July 2019).

12. Bohlmann, F.; Zdero, C.; King, R.M.; Robinson, H. Ein neues germacran-8.12-olid und neue diterpene aus Polymnia canadensis. Phytochemistry 1980, 19, 115-118. [CrossRef]

13. Kartesz, J.T. BONAP's North American Plant Atlas. Available online: http://bonap.net/Napa/TaxonMaps/ Genus/County/Rudbeckia (accessed on 17 July 2019).

14. Missouri Botanical Garden Tropicos.org. Available online: www.tropicos.org (accessed on 21 December 2019).

15. Weakley, A.S. Flora of the Southern and Mid-Atlantic States. Available online: http://www.herbarium.unc. edu/flora.htm (accessed on 17 July 2019).

16. Kartesz, J.T. BONAP's North American Plant Atlas. Available online: http://bonap.net/Napa/TaxonMaps/ Genus/County/Silphium (accessed on 17 July 2019).

17. Kowalski, R. Secondary metabolites in Silphium integrifolium in the first 2 years of cultivation. N. Z. J. Crop Hortic. Sci. 2004, 32, 397-406. [CrossRef]

18. Kartesz, J.T. BONAP's North American Plant Atlas. Available online: http://bonap.net/Napa/TaxonMaps/ Genus/County/Smallanthus (accessed on 17 July 2019).

19. Kartesz, J.T. BONAP's North American Plant Atlas. Available online: http://bonap.net/Napa/TaxonMaps/ Genus/County/Solidago (accessed on 17 July 2019).

20. Reznicek, G.; Jurenitsch, J.; Plasun, M.; Korhammer, S.; Haslinger, E.; Hiller, K.; Kubelka, W. Four major saponins from Solidago canadensis. Phytochemistry 1991, 30, 1629-1633. [CrossRef] 
21. Reznicek, G.; Jurenitsch, J.; Freiler, M.; Korhammer, S.; Haslinger, E.; Hiller, K.; Kubelka, W. Isolation and structure elucidation of further new saponins from Solidago canadensis. Planta Med. 1992, 58, 94-98. [CrossRef]

22. Apáti, P.; Szentmihályi, K.; Balázs, A.; Baumann, D.; Hamburger, M.; Kristó, T.S.; Szőke, É.; Kéry, Á. HPLC Analysis of the flavonoids in pharmaceutical preparations from Canadian goldenrod (Solidago canadensis). Chromatographia 2002, 56, S65-S68. [CrossRef]

23. Wu, B.; Takahashi, T.; Kashiwagi, T.; Tebayashi, S.; Kim, C.-S. New flavonoid glycosides from the leaves of Solidago altissima. Chem. Pharm. Bull. 2007, 55, 815-816. [CrossRef]

24. Radusiene, J.; Marska, M.; Ivanauskas, L.; Jakstas, V.; Karpaviciene, B. Assessment of phenolic compound accumulation in two widespread goldenrods. Ind. Crops Prod. 2015, 63, 158-166. [CrossRef]

25. Ichihara, K.I.; Kawar, T.; Kaji, M.; Noda, M. A new polyacetylene from Solidago altissima L. Agric. Biol. Chem. 1976, 40, 353-358. [CrossRef]

26. Ichihara, K.I.; Kawai, T.; Noda, M. Polyacetylenes of Solidago altissima L. Agric. Biol. Chem. 1978, 42, $427-431$. [CrossRef]

27. Tori, M.; Katto, A.; Sono, M. Nine new clerodane diterpenoids from rhizomes of Solidago altissima. Phytochemistry 1999, 52, 487-493. [CrossRef]

28. Chaturvedula, V.S.P.; Zhou, B.N.; Gao, Z.; Thomas, S.J.; Hecht, S.M.; Kingston, D.G.I. New lupane triterpenoids from Solidago canadensis that inhibit the lyase activity of DNA polymerase $\beta$. Bioorg. Med. Chem. 2004, 12, 6271-6275. [CrossRef]

29. Kartesz, J.T. BONAP's North American Plant Atlas. Available online: http://bonap.net/Napa/TaxonMaps/ Genus/County/Xanthium (accessed on 17 July 2019).

30. Kamboj, A.; Saluja, A.K. Phytopharmacological review of Xanthium strumarium L. (Cocklebur). Int. J. Green Pharm. 2010, 4, 129-139. [CrossRef]

31. Satyal, P.; Craft, J.D.; Dosoky, N.S.; Setzer, W.N. The chemical compositions of the volatile oils of garlic (Allium sativum) and wild garlic (Allium vineale). Foods 2017, 6, 63. [CrossRef]

32. Davé, P.C.; Vogler, B.; Setzer, W.N. Chemical compositions of the leaf essential oils of Aralia spinosa from three habitats in Northern Alabama. Am. J. Plant Sci. 2011, 2, 507-510. [CrossRef]

33. Eiter, L.C.; Fadamiro, H.; Setzer, W.N. Seasonal variation in the leaf essential oil composition of Zanthoxylum clava-herculis growing in Huntsville, Alabama. Nat. Prod. Commun. 2010, 5, 457-460. [CrossRef]

34. Steinberg, K.M.; Satyal, P.; Setzer, W.N. Bark essential oils of Zanthoxylum clava-herculis and Ptelea trifoliata: Enantiomeric distribution of monoterpenoids. Nat. Prod. Commun. 2017, 12, 961-963. [CrossRef]

35. Woods, K.E.; Chhetri, B.K.; Jones, C.D.; Goel, N.; Setzer, W.N. Bioactivities and compositions of Betula nigra essential oils. J. Med. Act. Plants 2013, 2, 1-9.

36. Stewart, C.D.; Jones, C.D.; Setzer, W.N. Essential oil compositions of Juniperus virginiana and Pinus virginiana, two important trees in Cherokee traditional medicine. Am. J. Essent. Oils Nat. Prod. 2014, 2, 17-24.

37. Setzer, W.N. Chemical composition of the leaf essential oil of Lindera benzoin growing in North Alabama. Am. J. Essent. Oils Nat. Prod. 2016, 4, 1-3.

38. Mekala, A.B.; Satyal, P.; Setzer, W.N. Phytochemicals from the bark of Rhamnus caroliniana. Nat. Prod. Commun. 2017, 12, 403-406. [CrossRef]

39. Kaler, K.M.; Setzer, W.N. Seasonal variation in the leaf essential oil composition of Sassafras albidum. Nat. Prod. Commun. 2008, 3, 829-832. [CrossRef]

40. Kennedy, J.E.; Davé, P.C.; Harbin, L.N.; Setzer, W.N. Allelopathic potential of Sassafras albidum and Pinus taeda essential oils. Allelopath. J. 2011, 27, 111-122.

41. Craft, J.D.; Setzer, W.N. Leaf essential oil composition of Tsuga canadensis growing wild in North Alabama and Northwest Georgia. Am. J. Essent. Oils Nat. Prod. 2017, 5, 26-29.

42. Lopez, E.M.; Craft, J.D.; Setzer, W.N. Volatile composition of Vicia caroliniana growing in Huntsville, Alabama. Am. J. Essent. Oils Nat. Prod. 2017, 5, 8-10.

43. Stewart, C.D.; Jones, C.D.; Setzer, W.N. Leaf essential oil compositions of Rudbeckia fulgida Aiton, Rudbeckia hirta L., and Symphyotrichum novae-angliae (L.) G.L. Nesom (Asteraceae). Am. J. Essent. Oils Nat. Prod. 2014, 2, 36-38.

44. Lawson, S.K.; Sharp, L.G.; Powers, C.N.; McFeeters, R.L.; Satyal, P.; Setzer, W.N. Essential oil compositions and antifungal activity of sunflower (Helianthus) species growing in north Alabama. Appl. Sci. 2019, 9, 3179. [CrossRef] 
45. Craft, J.D.; Lawson, S.K.; Setzer, W.N. Leaf essential oil compositions of bear's foot, Smallanthus uvedalia and Polymnia canadensis. Am. J. Essent. Oils Nat. Prod. 2019, 7, 31-35.

46. Liu, P.-Y.; Liu, D.; Li, W.-H.; Zhao, T.; Sauriol, F.; Gu, Y.-C.; Shi, Q.-W.; Zhang, M.-L. Chemical constituents of plants from the genus Eupatorium (1904-2014). Chem. Biodivers. 2015, 12, 1481-1515. [CrossRef] [PubMed]

47. Padalia, R.C.; Bisht, D.S.; Joshi, S.C.; Mathela, C.S. Chemical composition of the essential oil from Eupatorium adenophorum Spreng. J. Essent. Oil Res. 2009, 21, 522-524. [CrossRef]

48. Kurade, N.P.; Jaitak, V.; Kaul, V.K.; Sharma, O.P. Chemical composition and antibacterial activity of essential oils of Lantana camara, Ageratum houstonianum and Eupatorium adenophorum. Pharm. Biol. 2010, 48, 539-544. [CrossRef]

49. Pandey, A.K.; Mohan, M.; Singh, P.; Palni, U.T.; Tripathi, N.N. Chemical composition, antibacterial and antioxidant activity of essential oil of Eupatorium adenophorum Spreng. from Eastern Uttar Pradesh, India. Food Biosci. 2014, 7, 80-87. [CrossRef]

50. Ahluwalia, V.; Sisodia, R.; Walia, S.; Sati, O.P.; Kumar, J.; Kundu, A. Chemical analysis of essential oils of Eupatorium adenophorum and their antimicrobial, antioxidant and phytotoxic properties. J. Pest Sci. 2014, 87, 341-349. [CrossRef]

51. Maia, J.G.S.; Zoghbi, M.D.G.B.; Andrade, E.H.A.; Da Silva, M.H.L.; Luz, A.I.R.; Da Silva, J.D. Essential oils composition of Eupatorium species growing wild in the Amazon. Biochem. Syst. Ecol. 2002, 30, 1071-1077. [CrossRef]

52. Zygadlo, J.A.; Maestri, D.M.; Guzmán, C.A. Comparative study of the essential oils from three species of Eupatorium. Flavour Fragr. J. 1996, 11, 153-155. [CrossRef]

53. Zygadlo, J.A.; Lamarque, A.L.; Grosso, N.R.; Ariza Espinar, L. Analysis of the essential oil of the leaves of Eupatorium arnottianum Griseb. J. Essent. Oil Res. 1995, 7, 677-678. [CrossRef]

54. García, C.C.; Acosta, E.G.; Carro, A.C.; Belmonte, M.C.F.; Bomben, R.; Duschatzky, C.B.; Perotti, M.; Schuff, C.; Damonte, E.B. Virucidal activity and chemical composition of essential oils from aromatic plants of central west Argentina. Nat. Prod. Commun. 2010, 5, 1307-1310. [CrossRef]

55. Lancelle, H.G.; Giordano, O.S.; Sosa, M.E.; Tonn, C.E. Chemical composition of four essential oils from Eupatorium spp. Biological activities toward Tribolium castaneum (Coleoptera: Tenebrionidae). Rev. Soc. Entomol. Argent. 2009, 68, 329-338.

56. Albuquerque, M.R.J.R.; Souza, E.B.D.; Mesquita, E.F.; Nunes, E.P.; Cunha, A.N.; Silveira, E.R. Volatile constituents from leaves of Vernonia chalybaea Mart. and Eupatorium ballotaefolium H.B.K. J. Essent. Oil Res. 2001, 13, 376-377. [CrossRef]

57. Albuquerque, M.R.; Silveira, E.R.; De AUchôa, D.E.; Lemos, T.L.; Souza, E.B.; Santiago, G.M.; Pessoa, O.D. Chemical composition and larvicidal activity of the essential oils from Eupatorium betonicaeforme (D.C.) Baker (Asteraceae). J. Agric. Food Chem. 2004, 52, 6708-6711. [CrossRef] [PubMed]

58. Lorenzo, D.; Paz, D.; Davies, P.; Villamil, J.; Vila, R.; Cañigueral, S.; Dellacassa, E. Application of multidimensional gas chromatography to the enantioselective characterisation of the essential oil of Eupatorium buniifolium Hooker et Arnott. Phytochem. Anal. 2005, 16, 39-44. [CrossRef] [PubMed]

59. Umpiérrez, M.L.; Santos, E.; Mendoza, Y.; Altesor, P.; Rossini, C. Essential oil from Eupatorium buniifolium leaves as potential varroacide. Parasitol. Res. 2013, 112, 3389-3400. [CrossRef]

60. Senatore, F.; De Fusco, R.; Napolitano, F. Eupatorium cannabinum L. ssp. cannabinum (Asteraceae) essential oil: Chemical composition and antibacterial activity. J. Essent. Oil Res. 2001, 13, 463-466. [CrossRef]

61. Flamini, G.; Cioni, P.L.; Morelli, I. Analysis of the essential oil of the leaves and flowers/fruits of Eupatorium cannabinum L. from south Tuscany (central Italy). J. Essent. Oil Res. 2003, 15, 127-129. [CrossRef]

62. Paolini, J.; Costa, J.; Bernardini, A.-F. Analysis of the essential oil from aerial parts of Eupatorium cannabinum subsp. corsicum (L.) by gas chromatography with electron impact and chemical ionization mass spectrometry. J. Chromatogr. A 2005, 1076, 170-178. [CrossRef]

63. Morteza-Semnani, K.; Akbarzadeh, M.; Moshiri, K. The essential oil composition of Eupatorium cannabinum L. from Iran. Flavour Fragr. J. 2006, 21, 521-523. [CrossRef]

64. Judzentiene, A. Chemical composition of leaf and inflorescence essential oils of Eupatorium cannabinum L. from eastern Lithuania. J. Essent. Oil Res. 2007, 19, 403-406. [CrossRef]

65. Pino, J.A.; Rosado, A.; Fuentes, V. Essential oil of Eupatorium capillifolum (Lam.) Small from Cuba. J. Essent. Oil Res. 1998, 10, 79-80. [CrossRef] 
66. Tabanca, N.; Bernier, U.R.; Tsikolia, M.; Becnel, J.J.; Sampson, B.; Werle, C.; Demirci, B.; Baser, K.H.C.; Blythe, E.K.; Pounders, C.; et al. Eupatorium capillifolium essential oil: Chemical composition, antifungal activity, and insecticidal activity. Nat. Prod. Commun. 2010, 5, 1409-1415. [CrossRef]

67. Pimienta-Ramírez, L.; García-Rodríguez, Y.M.; Ríos-Ramírez, E.M.; Lindig-Cisneros, R.; Espinosa-García, F.J. Chemical composition and evaluation of the essential oil from Eupatorium glabratum as biopesticide against Sitophilus zeamais and several stored maize fungi. J. Essent. Oil Res. 2016, 28, 113-120. [CrossRef]

68. Schossler, P.; Schneider, G.L.; Wunsch, D.; Soares, G.L.G.; Zini, C.A. Volatile compounds of Baccharis punctulata, Baccharis dracunculifolia and Eupatorium laevigatum obtained using solid phase microextraction and hydrodistillation. J. Braz. Chem. Soc. 2009, 20, 277-287. [CrossRef]

69. Pisutthanan, N.; Liawruangrath, B.; Liawruangrath, S.; Baramee, A.; Apisariyakul, A.; Korth, J.; Bremner, J.B. Constituents of the essential oil from aerial parts of Chromolaena odorata from Thailand. Nat. Prod. Res. 2006, 20, 636-640. [CrossRef] [PubMed]

70. Owolabi, M.S.; Ogundajo, A.; Yusuf, K.O.; Lajide, L.; Villanueva, H.E.; Tuten, J.A.; Setzer, W.N. Chemical composition and bioactivity of the essential oil of Chromolaena odorata from Nigeria. Rec. Nat. Prod. 2010, 4, 72-78.

71. Joshi, R.K. Chemical composition of the essential oils of aerial parts and flowers of Chromolaena odorata (L.) R. M. King \& H. Rob. from Western Ghats region of north west Karnataka, India. J. Essent. Oil-Bear. Plants 2013, $16,71-75$.

72. Gupta, D.; Charles, R.; Garg, S.N. Chemical examination of the essential oil from the leaves of Eupatorium triplinerve Vahl. J. Essent. oil Res. 2004, 16, 473-475. [CrossRef]

73. Moldovan, Z.; Buleandră, M.; Oprea, E.; Mînea, Z. Studies on chemical composition and antioxidant activity of Rudbeckia triloba. J. Anal. Methods Chem. 2017, 2017, 3407312. [CrossRef]

74. Kowalski, R.; Wierciński, J.; Mardarowicz, M. Essential oil in leaves and inflorescences of Silphium integrifolium Michx. J. Essent. Oil Res. 2005, 17, 220-222. [CrossRef]

75. Kowalski, R. The chemical composition of essential oils and lipophilic extracts of Silphium integrifolium Michx. and S. trifoliatum L. leaves. Flavour Fragr. J. 2008, 23, 164-171. [CrossRef]

76. Kowalski, R. Antimicrobial activity of essential oils and extracts of rosinweed (Silphium trifoliatum and Silphium integrifolium) plants used by the American Indians. Flavour Fragr. J. 2008, 23, 426-433. [CrossRef]

77. Kowalski, R. Chemical composition of essential oils and lipophilic extracts of Silphium integrifolium and S. trifoliatum inflorescences. Chem. Nat. Compd. 2008, 44, 241-244. [CrossRef]

78. Kowalski, R. The chemical composition of essential oils and lipophilic extracts of Silphium integrifolium Michx. and Silphium trifoliatum L. rhizomes. J. Essent. Oil Res. 2008, 20, 255-259. [CrossRef]

79. Cicció, J.F. Composition of the essential oil from leaves of Smallanthus maculatus (Cav.) H. Rob. (Asteraceae). J. Essent. Oil Res. 2004, 16, 353-355. [CrossRef]

80. Chaverri, C.; Cicció, J.F. Composition of the essential oil from leaves of Smallanthus quichensis (Asteraceae) from Costa Rica. Bol. Latinoam. Caribe Plantas Med. Aromat. 2015, 14, 355-363.

81. Li, J.; Liu, J.; Lan, H.; Zheng, M.; Rong, T. GC-MS analysis of the chemical constituents of the essential oil from the leaves of yacon (Smallanthus sonchifolia). Front. Agric. China 2009, 3, 40-42. [CrossRef]

82. Weyerstahl, P.; Marschall, H.; Christiansen, C.; Kalemba, D.; Góra, J. Constituents of the essential oil of Solidago canadensis ("goldenrod") from Poland-A correction. Planta Med. 1993, 59, 281-282. [CrossRef]

83. Schmidt, C.O.; Bouwmeester, H.J.; Bülow, N.; König, W.A. Isolation, characterization, and mechanistic studies of (-)- $\alpha$-gurjunene synthase from Solidago canadensis. Arch. Biochem. Biophys. 1999, 364, 167-177. [CrossRef]

84. Kasali, A.A.; Ekundayo, O.; Paul, C.; König, W.A. epi-Cubebanes from Solidago canadensis. Phytochemistry 2002, 59, 805-810. [CrossRef]

85. Vogler, B.; Setzer, W.N. Characterization of Natural Products. In Natural Products from Plants; Cseke, L.J., Kirakosyan, A., Kaufman, P.B., Warber, S.L., Duke, J.A., Brielmann, H., Eds.; CRC Press: Boca Raton, FL, USA, 2006; pp. 319-387. ISBN 978-0-8493-2976-0.

86. Chanotiya, C.S.; Yadav, A. Natural variability in enantiomeric composition of bioactive chiral terpenoids in the essential oil of Solidago canadensis L. from Uttarakhand, India. Nat. Prod. Commun. 2008, 3, 263-266. [CrossRef]

87. Mishra, D.; Joshi, S.; Sah, S.P.; Bischt, G. Chemical composition, analgesic and antimicrobial activity of Solidago canadensis essential oil from India. J. Pharm. Res. 2011, 44, 63-66. 
88. Grul'ova, D.; Baranova, B.; Ivanova, V.; de Martino, L.; Mancini, E.; de Feo, V. Composition and bio activity of essential oils of Solidago spp. and their impact on radish and garden cress. Allelopath. J. 2016, 39, 129-142.

89. Shelepova, O.; Vinogradova, Y.; Zaitchik, B.; Ruzhitsky, A.; Grygorieva, O.; Brindza, J. Constituents of the essential oil in Solidago canadensis L. from Eurasia. Slovak J. Food Sci. 2018, 12, 20-25. [CrossRef]

90. Elshafie, H.S.; Grul'ová, D.; Baranová, B.; Caputo, L.; De Martino, L.; Sedlák, V.; Camele, I.; De Feo, V. Antimicrobial activity and chemical composition of essential oil extracted from Solidago canadensis L. growing wild in Slovakia. Molecules 2019, 24, 1206. [CrossRef]

91. Benelli, G.; Pavela, R.; Cianfaglione, K.; Nagy, D.U.; Canale, A.; Maggi, F. Evaluation of two invasive plant invaders in Europe (Solidago canadensis and Solidago gigantea) as possible sources of botanical insecticides. J. Pest Sci. 2019, 92, 805-821. [CrossRef]

92. El-Sherei, M.; Khaleel, A.; Motaal, A.A.; Abd-Elbaki, P. Effect of seasonal variation on the composition of the essential oil of Solidago canadensis cultivated in Egypt. J. Essent. Oil-Bear. Plants 2014, 17, 891-898. [CrossRef]

93. Esmaeili, A.; Rustaiyan, A.; Akbari, M.T.; Moazami, N.; Masoudi, S.; Amiri, H. Composition of the essential oils of Xanthium strumarium L. and Cetaurea solstitialis L. from Iran. J. Essent. Oil Res. 2006, 18, 427-429. [CrossRef]

94. Sharifi-Rad, J.; Hoseini-Alfatemi, S.M.; Sharifi-Rad, M.; Sharifi-Rad, M.; Iriti, M.; Sharifi-Rad, M.; Sharifi-Rad, R.; Raeisi, S. Phytochemical compositions and biological activities of essential oil from Xanthium strumarium L. Molecules 2015, 20, 7034-7047. [CrossRef]

95. Parveen, Z.; Mazhar, S.; Siddique, S.; Manzoor, A.; Ali, Z. Chemical composition and antifungal activity of essential oil from Xanthium strumarium L. leaves. Indian J. Pharm. Sci. 2017, 79, 316-321. [CrossRef]

96. Jacyno, J.M.; Montemurro, N.; Bates, A.D.; Cutler, H.G. Phytotoxic and antimicrobial properties of cyclocolorenone from Magnolia grandiflora L. J. Agric. Food Chem. 1991, 39, 1166-1168. [CrossRef]

97. Schmidt, J.M.; Noletto, J.A.; Vogler, B.; Setzer, W.N. Abaco bush medicine: Chemical composition of the essential oils of four aromatic medicinal plants from Abaco Island, Bahamas. J. Herbs Spices Med. Plants 2006, 12, 43-65. [CrossRef]

98. Gardini, F.; Lanciotti, R.; Caccioni, D.R.L.; Guerzoni, M.E. Antifungal activity of hexanal as dependent on its vapor pressure. J. Agric. Food Chem. 1997, 45, 4297-4302. [CrossRef]

99. Gardini, F.; Lanciotti, R.; Guerzoni, M.E. Effect of trans-2-hexenal on the growth of Aspergillus flavus in relation to its concentration, temperature and water activity. Lett. Appl. Microbiol. 2001, 33, 50-55. [CrossRef] [PubMed]

100. Neri, F.; Mari, M.; Menniti, A.M.; Brigati, S. Activity of trans-2-hexenal against Penicillium expansum in “Conference" pears. J. Appl. Microbiol. 2006, 100, 1186-1193. [CrossRef]

101. Van Vuuren, S.F.; Viljoen, A.M. Antimicrobial activity of limonene enantiomers and 1,8-cineole alone and in combination. Flavour Fragr. J. 2007, 22, 540-544. [CrossRef]

102. Ma, B.; Ban, X.; Huang, B.; He, J.; Tian, J.; Zeng, H.; Chen, Y.; Wang, Y. Interference and mechanism of dill seed essential oil and contribution of carvone and limonene in preventing Sclerotinia rot of rapeseed. PLoS ONE 2015, 10, e0131733. [CrossRef]

103. Jing, L.; Lei, Z.; Li, L.; Xie, R.; Xi, W.; Guan, Y.; Sumner, L.W.; Zhou, Z. Antifungal activity of Citrus essential oils. J. Agric. Food Chem. 2014, 62, 3011-3033. [CrossRef]

104. DeCarlo, A.; Johnson, S.; Okeke-Agulu, K.I.; Dosoky, N.S.; Wax, S.J.; Owolabi, M.S.; Setzer, W.N. Compositional analysis of the essential oil of Boswellia dalzielii frankincense from West Africa reveals two major chemotypes. Phytochemistry 2019, 164, 24-32. [CrossRef]

105. Adams, R.P. Identification of Essential Oil Components by Gas Chromatography/Mass Spectrometry, 4th ed.; Allured Publishing: Carol Stream, IL, USA, 2007.

106. NIST17. NIST17; National Institute of Standards and Technology: Gaithersburg, MD, USA, 2017.

107. Mondello, L. FFNSC 3; Shimadzu Scientific Instruments: Columbia, MD, USA, 2016.

108. Satyal, P. Development of GC-MS Database of Essential Oil Components by the Analysis of Natural Essential Oils and Synthetic Compounds and Discovery of Biologically Active Novel Chemotypes in Essential Oils. Ph.D. Thesis, University of Alabama in Huntsville, Huntsville, AL, USA, 2015.

109. Powers, C.N.; Osier, J.L.; McFeeters, R.L.; Brazell, C.B.; Olsen, E.L.; Moriarity, D.M.; Satyal, P.; Setzer, W.N. Antifungal and cytotoxic activities of sixty commercially-available essential oils. Molecules 2018, 23, 1549. [CrossRef]

(C) 2020 by the authors. Licensee MDPI, Basel, Switzerland. This article is an open access article distributed under the terms and conditions of the Creative Commons Attribution (CC BY) license (http://creativecommons.org/licenses/by/4.0/). 